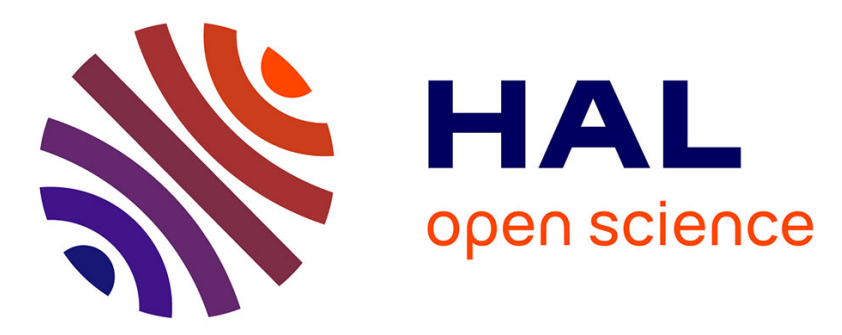

\title{
Centres de production et diffusion des silex bédouliens au Chasséen
}

\author{
Vanessa Léa
}

\section{To cite this version:}

Vanessa Léa. Centres de production et diffusion des silex bédouliens au Chasséen. Gallia Préhistoire - Archéologie de la France préhistorique, 2004, 46, pp.231-250. 10.3406/galip.2004.2044 . hal00344444

\section{HAL Id: hal-00344444 \\ https://hal.science/hal-00344444}

Submitted on 4 Dec 2008

HAL is a multi-disciplinary open access archive for the deposit and dissemination of scientific research documents, whether they are published or not. The documents may come from teaching and research institutions in France or abroad, or from public or private research centers.
L'archive ouverte pluridisciplinaire HAL, est destinée au dépôt et à la diffusion de documents scientifiques de niveau recherche, publiés ou non, émanant des établissements d'enseignement et de recherche français ou étrangers, des laboratoires publics ou privés.

\section{(이) $\$$}

Distributed under a Creative Commons Attribution - NonCommercial - NoDerivatives| 4.0 


\title{
CENTRES DE PRODUCTION ET DIFFUSION DES SILEX BÉDOULIENS AU CHASSÉEN
}

\author{
Vanessa LÉA*
}

\begin{abstract}
Mots-clés. Chasséen méridional, Vaucluse, silex bédouliens, ateliers de production, diffusion.
Résumé. $A u x V^{e}$ et $I V^{e}$ millénaires avant J.-C., en contexte chasséen, la diffusion des silex bédouliens de Haute-Provence (Crétacé inférieur) atteint une rare ampleur et constitue un phénomène tout à fait original. En effet, l'essentiel des assemblages lithiques est composé de productions effectuées à partir de cette matière première exportée. Or, si les études concernant les sites récepteurs ou consommateurs ont été nombreuses ces dernières années, les contextes de production restent à l'heure actuelle totalement méconnus. De récentes découvertes dans la zone de production relancent la recherche sur ce thème indispensable à une meilleure appréhension des sociétés chasséennes. L'approche de deux nouveaux ateliers de production, Les Trois Termes (Gordes) et Rocalibert (Piolenc), permet d'aborder le renforcement des spécialisations artisanales et les contextes de production au Chasséen.
\end{abstract}

Keys-words. Chassey culture, Vaucluse (region), Bedoulian flint, production centres, diffusion.

Abstract. At the $5^{\text {th }}$ and $4^{\text {th }}$ millenium BC, in a Chassean context, the distribution of Bedoulian flint of Haute-Provence (Lower Cretaceous), extents impressively and is considered as an absolutely original phenomenon. The lithic assemblages are mainly composed of products made from this raw material being exported. But if numerous studies dealing with consumer sites have been undergone these last years, the production environment is still totally misunderstood. Recent discoveries in the production area give a new impetus to research on this essential subject for a better understanding of Chassean communities. The approach of two new production sites, Les Trois Termes (Gordes) and Rocalibert (Piolenc) gives the opportunity to study the increasing specializations and the context of production during the Chassean.

Palabras claves. Chasense meridional, Vaucluse, sílex bedoulienses, talleres de producción, difusión.

Resumen. En el $V^{o}$ y en el IV milenario a. C., en contexto chasense, la difusión de los sílex bedoulienses de Haute-Provence (Cretácico inferior) alcanza una amplitud excepcional, lo cual constituye un fenómeno de lo más original. En efecto, la mayor parte de los conjuntos líticos está compuesta de producciones realizadas a partir de esta materia prima de importación. Sin embargo, si bien fueron numerosos en los últimos años los estudios acerca de los sitios receptores o consumadores, los contextos de producción siguen hasta ahora totalmente desconocidos. Hallazgos recientes en la zona de producción enfocan de nuevo las investigaciones en este tema imprescindible para lograr una mejor comprensión de las sociedades chasenses. Por ende, el examen de dos nuevos talleres de producción - los Trois Termes (Gordes) y Rocalibert (Piolenc) - permite plantear el carácter cada vez más fuerte de las especializaciones artesanales y los contextos de producción en el Chasense.

\section{PROBLÉMATIQUE}

Aux $V^{\mathrm{e}}$ et $\mathrm{IV}^{\mathrm{e}}$ millénaires avant J.-C., la diffusion des silex bédouliens de Haute-Provence (Crétacé inférieur; Binder, 1998) atteint une rare ampleur et constitue un phénomène tout à fait original. Cette originalité se mesure du point de vue de l'étendue géographique des réseaux (Midi de la France, Catalogne à l'ouest - Suisse, Piémont-Ligurie à l'est), des quantités de matières premières, et de la diversité des productions exportées (préformes chauffées prêtes à être

* CÉPAM (UMR 6130 du CNRS), 250 avenue Albert-Einstein, Sophia-Antipolis, F-06560 Valbonne, et ATER Université de Toulouse-Le-Mirail. 
débitées, produits finis dont des lames robustes en silex non chauffé, éclats). L'étude de ces réseaux révèle une segmentation de la chaîne opératoire dans le temps et dans l'espace, en correspondance avec une différenciation des savoir-faire : les très délicates phases de mise en forme ont lieu sur les sites producteurs à proximité des affleurements et seraient l'œuvre de spécialistes, alors que sur les sites consommateurs, le débitage de lamelles correspondrait à une production domestique rendue aisée grâce au traitement thermique des préformes (Binder, 1984, 1991 ; Binder, Perlès, 1990).

Paradoxalement, les études lithiques ont jusqu'à présent presque exclusivement concerné les sites consommateurs (Binder, 1984, 1991 ; Binder, Gassin, 1988 ; Briois, 1997 ; Briois et al., 1998 ; Gassin, 1996 ; Léa, 1997, 2002, 2003a, 2003b, 2004 ; Léa et al., 2004a ; Vaquer, 1990). Un récent programme de recensement a néanmoins été réalisé afin de définir un cadre général des gîtes de matières premières disponibles et d'évaluer l'aptitude à la taille des différents types de silex bédouliens (Binder et collab., 1998). Mais force est d'avouer que les données ayant trait aux ateliers de mise en forme sont éparses, inédites et dans la plupart des cas issues de fouilles anciennes (Léa et al., 2004b). On peut citer pour mémoire les fouilles de l'abri du Levant de Léaunier à Malaucène (Catelan, Catelan, 1922a, 1922b ; Barthélemy, 1952-1956), de l'abri Grangeon à Veaux (Vincent, inédit ; Courtin, 1974, p. 61) et celles de Schmid à Veaux-Malaucène (Schmid, 1962), sans oublier de nombreux ramassages de surface réalisés par des amateurs. Or, si ces recherches ont révélé l'envergure des travaux réalisés par les carriers néolithiques, aucune étude approfondie n'a été faite à ce jour. En effet, les centres de production ne peuvent pas facilement être abordés pour au moins deux raisons : d'une part l'ampleur considérable des tas de déblais pose de sérieux problèmes logistiques, et d'autre part la présence d'exploitations s'inscrivant dans la diachronie (du Paléolithique jusqu'au $\mathrm{xx}^{\mathrm{e}}$ siècle avec les ateliers de pierres à fusils) rend plus difficile l'identification d'ensembles homogènes du Chasséen (Léa et al., 2004b).

À l'heure actuelle, nous ne connaissons donc rien des modalités de fabrication des productions en silex bédouliens vouées à l'exportation. L'étude de l'organisation de la production est, à notre sens, pourtant fondamentale à l'appréhension des sociétés chasséennes et plus particulièrement à celle du renforcement des spécialisations artisanales. Cette approche se structure en quatre axes de recherche dont les principales questions peuvent être ainsi exposées.
A. Nature et caractérisation des productions: quelles sont les modalités d'extraction du silex, ainsi que les conditions de réalisation nécessaires au traitement thermique des préformes ? Par ailleurs, est-il possible de dire si à différents types de production correspondent différents ateliers ou si plusieurs types de production sont issus d'un même atelier?

B. Organisation spatiale de la production et des réseaux de diffusion: comment s'organise la production sur les sites de mise en forme? Existe-t-il, par exemple, un espace réservé pour les différentes étapes de la chaîne opératoire (extraction, mise en forme, traitement thermique, fin de mise en forme) à l'instar de ce qui est connu pour les ateliers de haches en pélites-quartz des Vosges (Pétrequin, Jeunesse, 1995 , p. 27) ? En ce qui concerne les réseaux de diffusion, la circulation des productions en silex bédouliens est-elle directe, des sites producteurs aux sites consommateurs, ou bien existe-t-il des sites intermédiaires qui joueraient le rôle de relais?

C. Évolution dans l'organisation des productions : l'organisation de la production est-elle la même pendant toute la durée du Chasséen ou peut-on observer des changements techniques? L'étude du fonctionnement des réseaux (Léa et al., 2004a) suggère en effet une évolution d'ordre quantitatif (hausse des quantités exportées au Chasséen récent) et qualitatif (passage d'un style semi-conique à un style quadrangulaire) (Binder, 1991) ; or, nous ne savons pas à quels changements dans l'organisation de la production cela correspond.

D. Organisation sociale de la production: cette approche s'intéresse à l'identité des populations qui exploitent les sources de matières premières (D1), au contexte de production (D2) ainsi qu'au savoir-faire mis en œuvre sur les centres producteurs (D3).

D1. Par qui sont exploitées ces sources de matières premières? Est-ce par une ou plusieurs populations?

D2. Dans quel contexte ces productions ont-elles été réalisées, autrement dit, s'agit-il d'un habitat temporaire ou bien d'un habitat permanent lié à l'exploitation des terroirs agro-pastoraux, par exemple ? Quelle est alors la part de la production réservée à l'usage domestique sur les ateliers? D3. Quels sont les savoir-faire mis en ouvre sur ce type de site ? Est-il, par exemple, possible d'identifier les témoins de phases d'apprentissage ou s'agit-il exclusivement de haut niveau de savoir-faire?

Nous n'avons évidemment pas la prétention de répondre ici à toutes ces questions qui doivent être traitées dans le cadre d'un programme de recherche d'envergure 
de longue durée. Cependant, deux récentes découvertes assez exceptionnelles apportent d'ores et déjà des informations nouvelles concernant les centres de production et la diffusion des silex bédouliens (fig. 1). Il s'agit de deux gisements vauclusiens qui se situent à proximité des affleurements, Les Trois Termes (Gordes) et Rocalibert (Piolenc). L'analyse technologique de ces assemblages lithiques n'est pour l'instant pas achevée, et ce ne sont que les résultats préliminaires que nous présentons ici. Il nous a néanmoins semblé important de faire part de ces découvertes qui offrent d'emblée certaines réponses aux questions posées ci-dessus.

\section{LES TROIS TERMES (GORDES) \\ PRÉSENTATION ET INTÉRÊT DU SITE}

Le site des Trois Termes prend place au bas du Pied Gaudin, dans le vallon de Ferrière, à la limite des communes de Murs et de Gordes (fig. 1). Découvert en 2000 par M. Castan à la suite d'un labour, il a fait l'objet de fréquents ramassages par son inventeur pendant deux années. Le matériel lithique est très abondant $(n=2043)$ et extrêmement concentré sur une surface de seulement quatre mètres carrés environ. Si le contexte archéologique n'est pour l'instant pas connu, l'exceptionnelle concentration du mobilier en surface, son homogénéité ainsi que la réalisation de nombreux remontages laissent supposer la présence d'une structure (une fosse?) arasée par le tracteur. L'industrie lithique taillée a été récoltée en même temps qu'une hache polie en roche verte et plusieurs éléments céramiques $(\mathrm{n}=108$, dont 14 bords, 3 cols, 2 carènes, 2 décors, 1 préhension). La céramique est de belle qualité, à pâte dense, avec des parois très régulières (Georjon, communication personnelle). Les trois formes reconstituées révèlent une coupe en calotte de sphère, une écuelle à carène basse, fond aplati, paroi verticale, bord éversé et aminci, ainsi qu'un vase à col présentant un léger ressaut interne au niveau de la rupture de pente qui n'est pas marquée à l'extérieur. Les décors concernent deux tessons. Le premier montre une ligne parallèle au bord, traitée en microsillon ou incisée; le second a une lèvre plate décorée de traits gravés ou en microsillons perpendiculaires au bord. Ces premières observations, sur un échantillon certes pour l'instant très réduit, font référence à un Chasséen récent C ou D (Beeching, 1995) de la moyenne vallée du Rhône (forme de l'écuelle carénée,

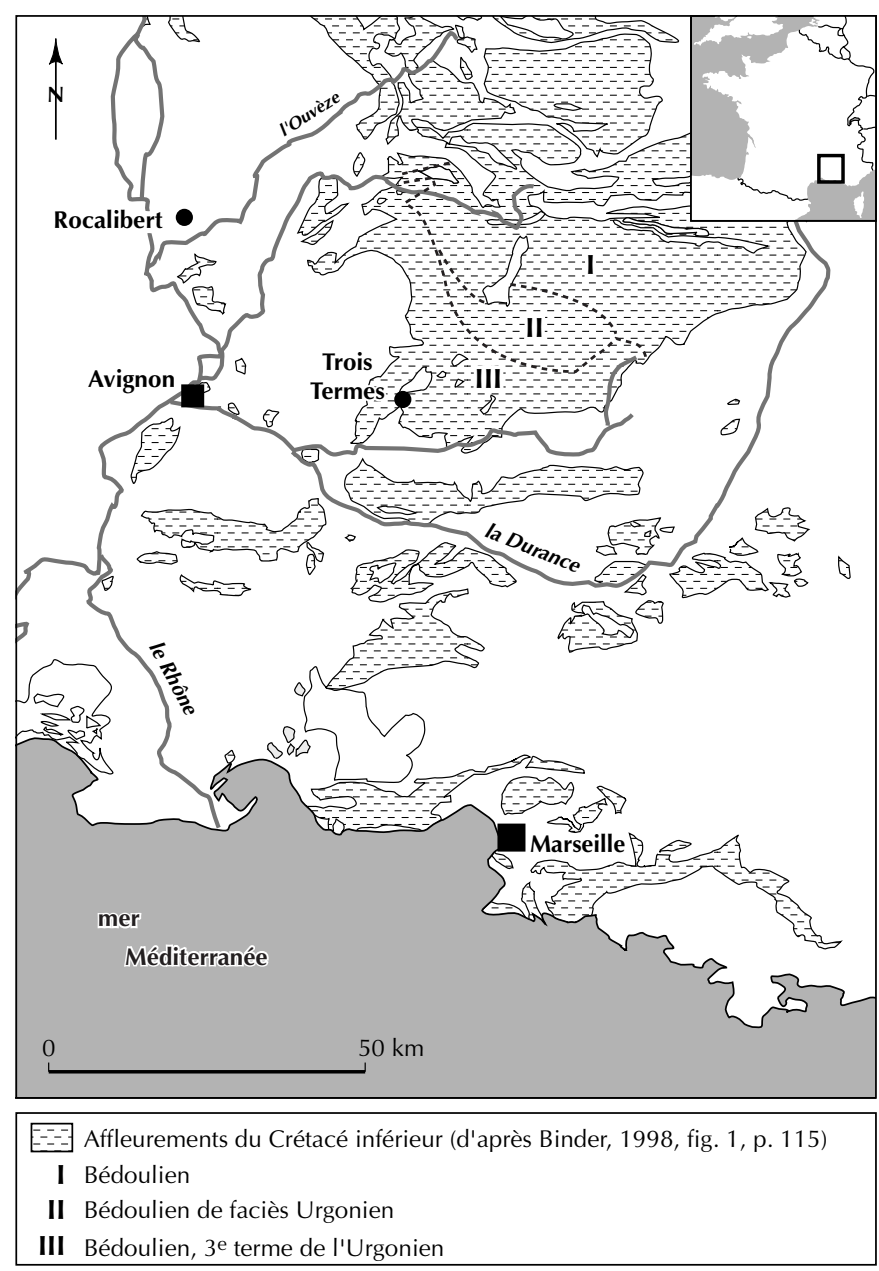

Fig. 1 - Situation géographique des sites des Trois Termes, Gordes et de Rocalibert, Piolenc (carte M. Gazenbeek).

lèvres plates et qualité des pâtes) (Georjon, communication personnelle).

À proximité, mais en dehors de l'aire de récolte très réduite, une meule ainsi qu'un maillet en quartz ont de même été trouvés. Or, la présence de maillets est ici très intéressante et doit être mise en relation avec les nombreux exemplaires déjà trouvés à Murs ou sur d'autres gîtes d'extraction des silex bédouliens (Deydier, 1904, 1905, 1907, 1908, 1911 ; Raymond, 1905 ; Moulin, 1905, 1908 ; Déchelette, 1908, p. 528 ; Carias, 1919 ; Cotte, 1924, t. II, p. 18-31 ; Vayson de Pradenne, 1923, 1934 ; Binder, 1998, p. 114 ; Léa et al., 2004b).

L'intérêt majeur du gisement des Trois Termes, situé à proximité immédiate des gîtes de matières premières, est d'offrir des informations concernant l'étude des phases de 


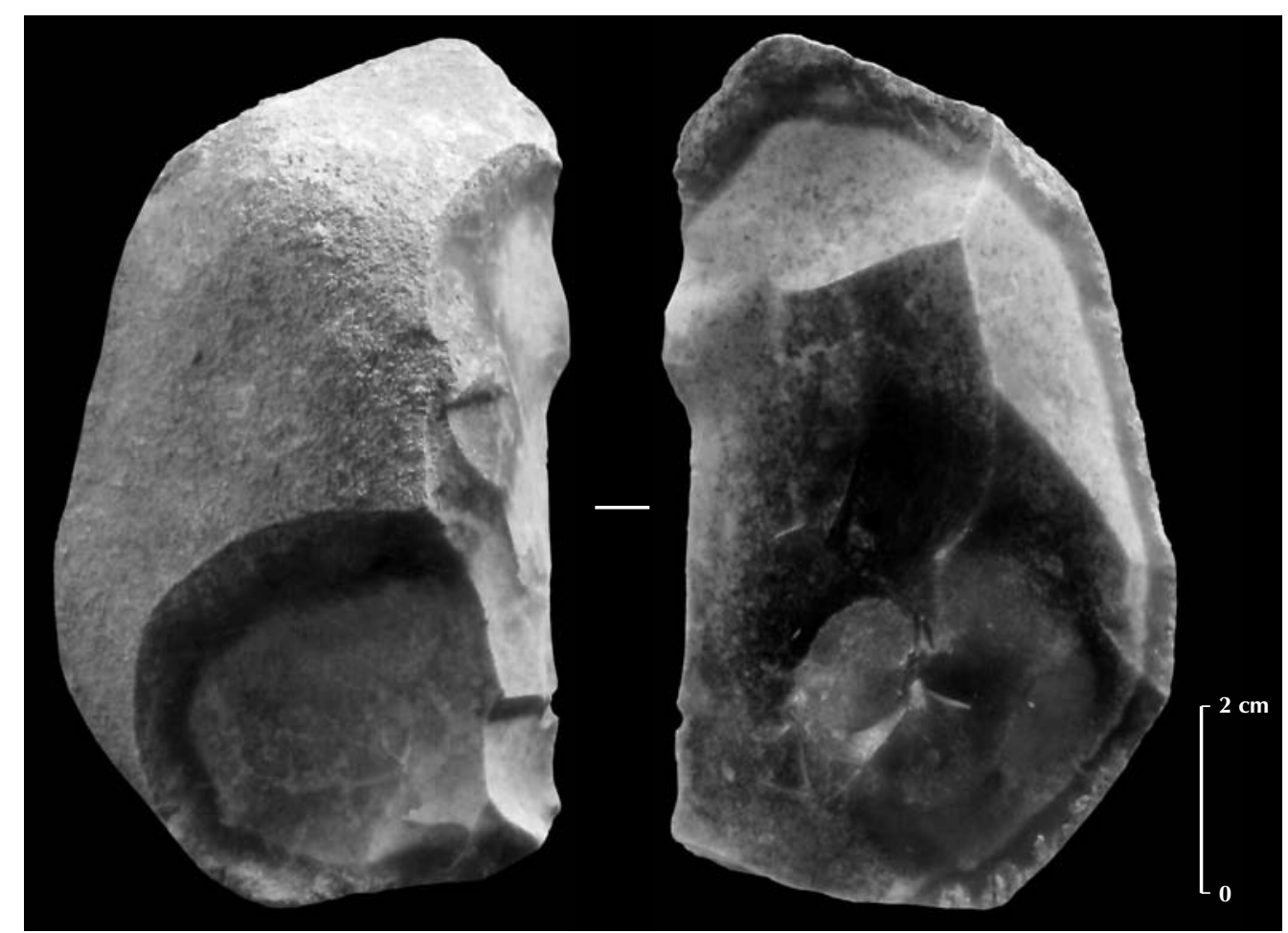

Fig. 2 - Les Trois Termes : éclat thermique.

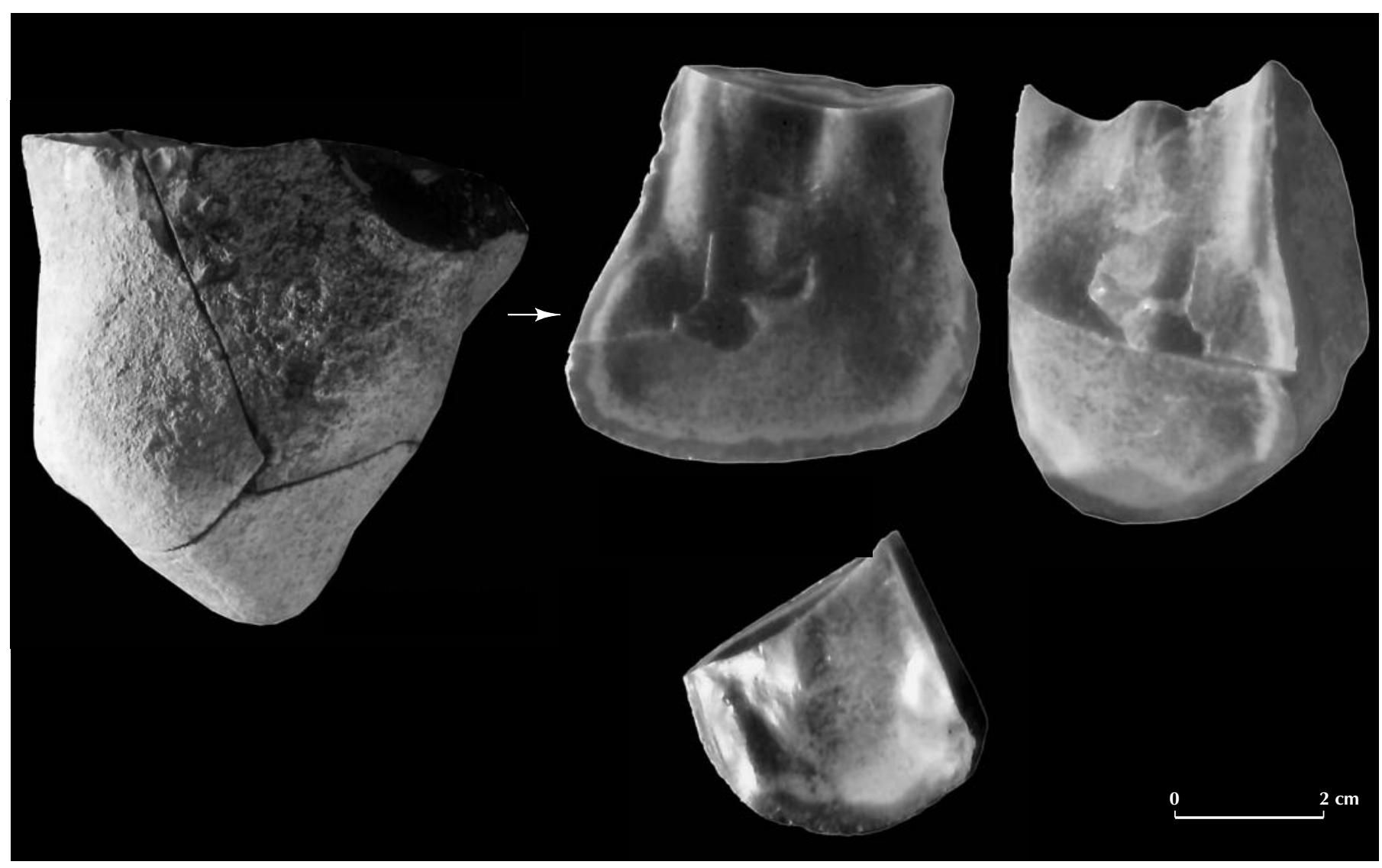

Fig. 3 - Les Trois Termes : remontage d'un bloc de silex bédoulien éclaté par le feu. 
mise en forme des préformes chauffées. Pour l'heure, nous ne connaissons pas exactement la morphologie des rognons bruts prélevés. La réalisation de prospections et de sondages systématiques sera donc pour cela nécessaire.

\section{LA RÉALISATION SUR PLACE DE LA MISE EN FORME ET DU TRAITEMENT THERMIQUE DES PRÉFORMES}

L'état de conservation du matériel lithique n'est pas très bon en raison de la présence d'une patine blanchâtre sur la plupart des pièces. L'assemblage récolté est composé d'éclats pour l'essentiel ( $\mathrm{n}=1538$, soit $75 \%$ de l'industrie), de produits laminaires $(\mathrm{n}=449$, soit $22 \%$ ) et de nucléus ( $\mathrm{n}=18)$ dont seulement sept attestent un débitage de lamelles par pression après chauffe $(0,3 \%)$. À ces éléments, il faut ajouter la présence originale de 38 fragments de blocs éclatés par le feu : ils comportent une ou plusieurs face(s) d'éclatement caractéristique(s) montrant à la fois des surfaces mates et des surfaces luisantes (Guilbert, 2001; fig. 2). Des remontages ont permis de reconstituer presque entièrement deux blocs de module assez réduit ${ }^{1}$ (fig. 3). Dans les deux cas, un ou plusieurs éclats avaient été débités avant chauffe. Ces deux blocs, ainsi que tous les éclats thermiques, correspondent sans doute à des ratés de chauffe: certains montrent clairement une chauffe non maîtrisée beaucoup trop importante (surfaces craquelées et accidentées). La présence aux Trois Termes de ces éléments est très intéressante car elle permet de poser l'hypothèse de la réalisation sur le site lui-même du traitement thermique des préformes. Celles-ci seraient donc chauffées sur les ateliers producteurs avant exportation. Ceci ne va pas dans le sens de ce qui avait été proposé à partir de l'étude de la série de la grotte de l'église supérieure, Var (Binder, Gassin, 1988, p. 121) où la présence de quelques éclats thermiques avait laissé supposer la pratique de la chauffe sur le site consommateur (Binder, communication orale). Nous étions cependant là en contexte Chasséen ancien (couches 7 et 8 de la grotte de l'église) et il se pourrait que le lieu de réalisation de la chauffe (sur les sites producteurs/sur les sites consommateurs) ne soit pas toujours le même durant toute la durée du Chasséen.

La mise en forme des préformes a été réalisée sur place. Elle a été opérée en partie avant traitement thermique, comme l'attestent les éclats en silex non chauffé corticaux

1. Hauteur : $6,5 \mathrm{~cm}$; largeur : $5,5 \mathrm{~cm}$; épaisseur : $4,5 \mathrm{~cm}$. (éclats d'entame ou à plages corticales résiduelles ; $\mathrm{n}=81$ ) ou non corticaux $(\mathrm{n}=74)$, et en partie après chauffe, comme en témoignent les éclats de mise en forme corticaux $(\mathrm{n}=465)$ ou non corticaux $(\mathrm{n}=918)$ ayant une face inférieure luisante. Ces derniers, les éclats non corticaux débités après chauffe, comportent, dans presque la moitié des cas, une ou plusieurs plages mates $(n=428)$. Leur face supérieure peut même être entièrement constituée de négatifs mats $(\mathrm{n}=94)$.

Parmi ces éclats de mise en forme, il faut souligner la surprenante quantité d'éclats se rapportant à l'ouverture du plan de pression après traitement thermique (près de 190 !). Ces éclats se caractérisent par une certaine variabilité du point de vue de leur morphologie et de leur module ${ }^{2}$. Une quarantaine se distingue par une forme plus allongée, révélant des nucléus assez cintrés (fig. 4 , n 3 et fig. $5, \mathrm{n}^{\text {os }} 1$ et 2 ). Certains des plus petits correspondent à une reprise du plan de pression en cours de débitage, mais il s'agit, dans la très grande majorité des cas, d'ouvertures après chauffe : il n'y a pas de négatifs de lamelles débitées par pression, et la face supérieure des éclats est constituée de plages mates. Ces éclats d'ouverture du plan de pression sont sans exception débités depuis la future surface de débitage. Deux options ont alors été observées : soit un seul éclat aménage la totalité de la surface du futur plan de pression (fig. $4, \mathrm{n}^{\circ} 3$ et fig. $5, \mathrm{n}^{\circ} 2$ ), soit deux éclats successifs sont nécessaires, comme le montrent plusieurs remontages (fig. 4, $\mathrm{n}^{\circ} 2$, fig. 5, $\mathrm{n}^{\circ} 1$, et fig. $6, \mathrm{n}^{\circ} 3$ ). Dans ce dernier cas, un premier éclat débordant à droite, précède un deuxième éclat débordant à gauche (fig. 6, no 3). Ces deux éclats portent donc sur leur bord abrupt, gauche ou droit, une partie des négatifs de préparation et/ou des résidus corticaux présents sur les flancs du nucléus.

L'observation de ces éclats permet de mieux situer l'intervention du traitement thermique au sein de la phase de mise en forme (fig. 7). À l'endroit où va prendre place le futur plan de pression, le cortex est presque entièrement enlevé avant la chauffe. Les résidus corticaux sur la face supérieure des éclats sont en effet peu nombreux (ils concernent plus souvent les bords latéraux des éclats, correspondant aux résidus présents sur les flancs du nucléus). Les préformes sont ensuite chauffées, et les éclats d'ouverture du plan de pression sont, sans exception ici,

2. Les plus grands et les plus épais mesurent jusqu'à $11 \mathrm{~cm}$ de longueur pour $5 \mathrm{~cm}$ de largeur; les plus petits environ $4,5 \mathrm{~cm}$ de longueur pour $2,5 \mathrm{~cm}$ de largeur. 


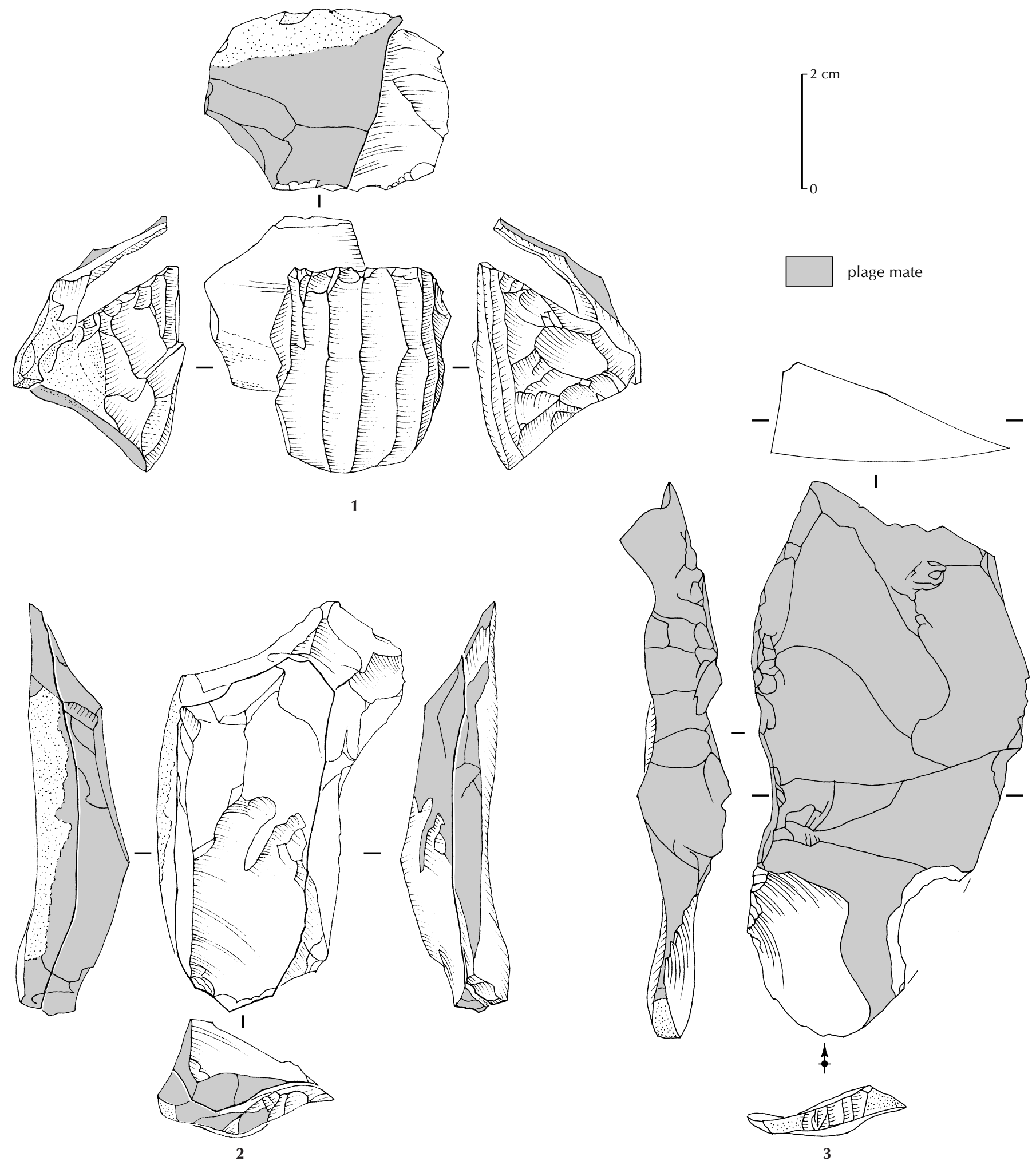

Fig. 4 - 1, Rocalibert : remontage d'une ouverture du plan de pression, présentant une face supérieure mate, sur son nucléus ; 2, 3, Les Trois Termes : ouvertures du plan de pression débitées après chauffe (dessin M. Grenet ; échelle 1/1). 


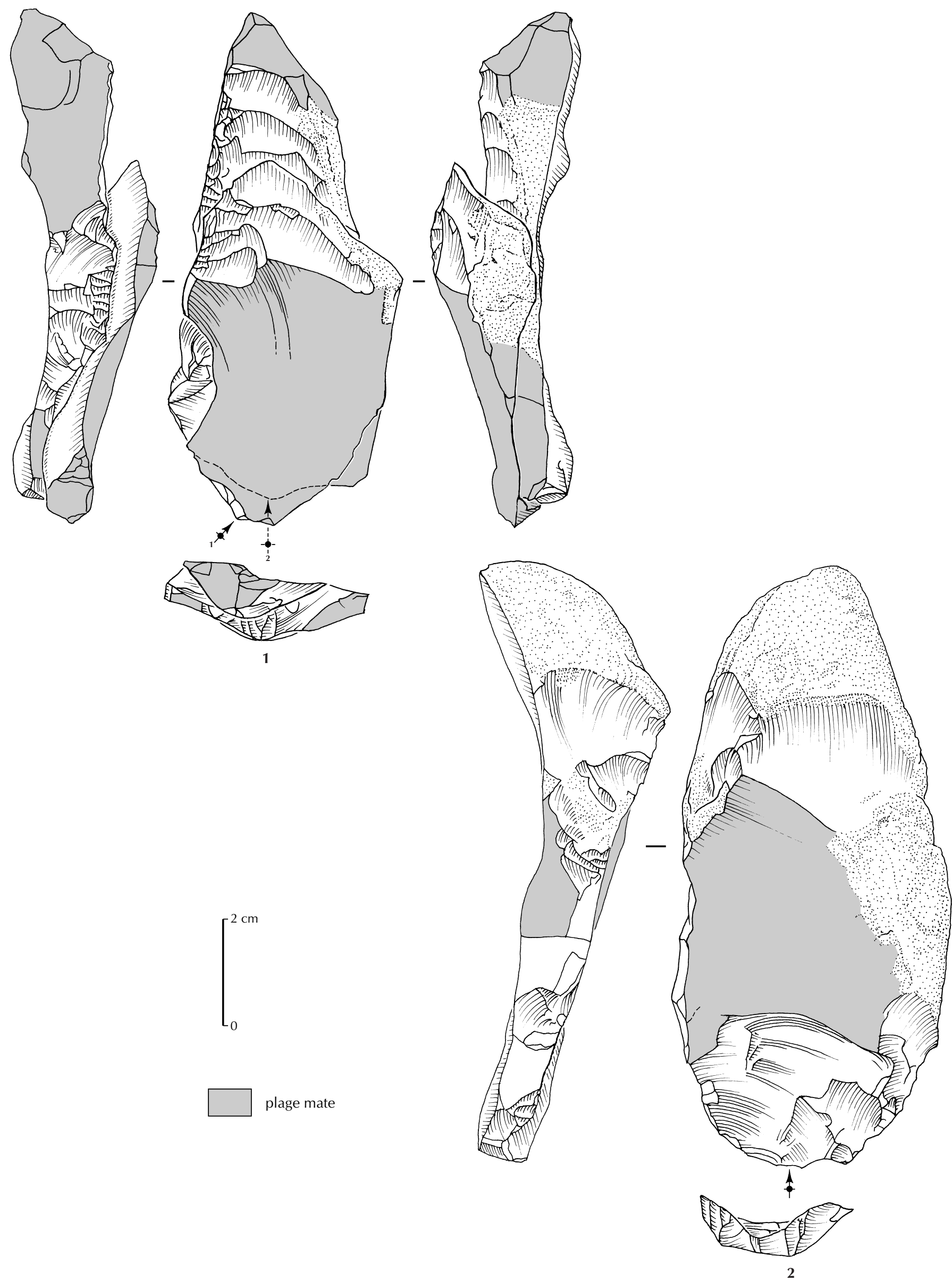

Fig. 5 - Les Trois Termes : 1, 2, ouvertures du plan de pression débitées après chauffe (dessin M. Grenet ; échelle 1/1). 

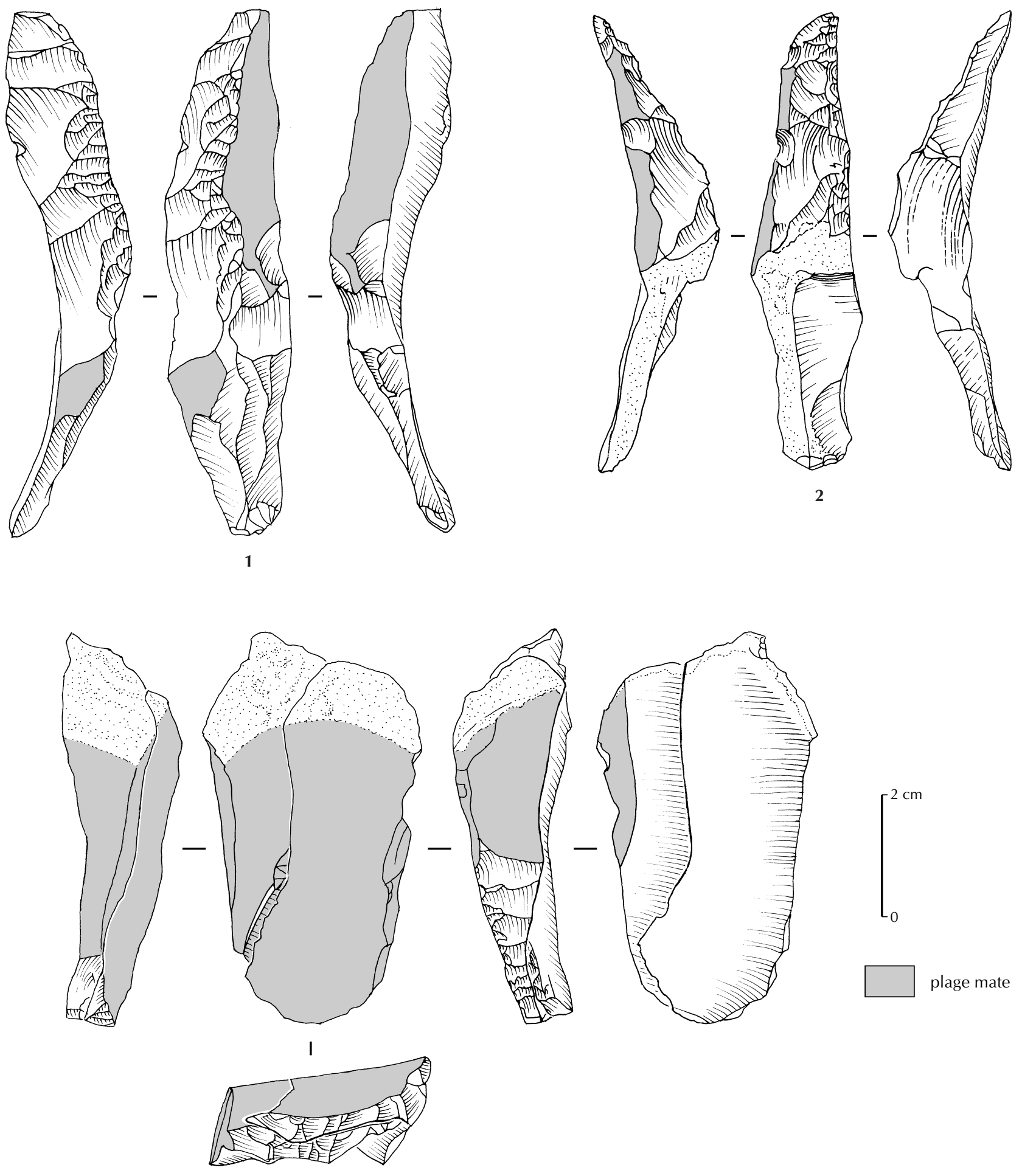

3

Fig. 6 - Les Trois Termes : 1, 2, lames à crête débitées après chauffe, présentant des traces de mise en forme avant et après traitement thermique; 3, ouverture du plan de pression grâce au débitage d'un éclat débordant à droite puis d'un éclat débordant à gauche (dessin M. Grenet ; échelle 1/1). 


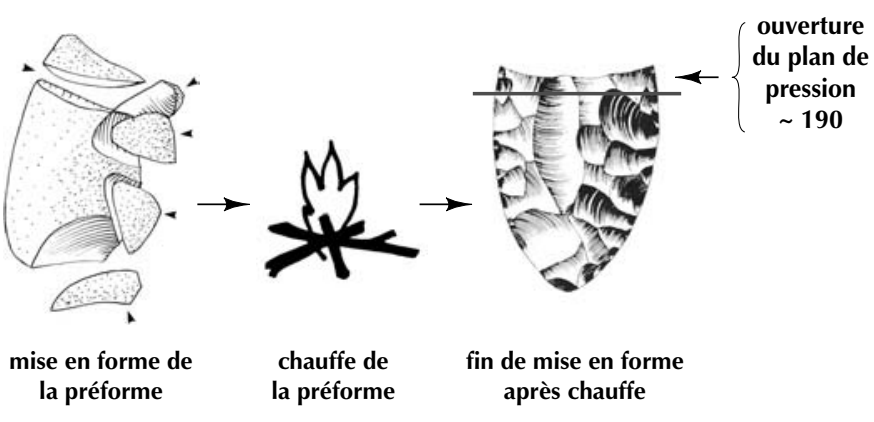

Fig. 7 - Situation du traitement thermique au sein de la chaîne opératoire sur le site des Trois Termes (vignettes tirées de Inizan et al., 1995, p. 40).

débités après chauffe : leur face supérieure est composée d'une ou plusieurs plages mates alors que leur face inférieure est luisante. Néanmoins, un remontage de trois éclats atteste un autre procédé : l'éclat d'entame et le deuxième éclat à résidus corticaux ont eux-mêmes été débités après traitement thermique.

Une première analyse des lames et des lamelles apporte, de même, des informations sur les modalités de mise en forme des préformes et sur les débuts du débitage laminaire. Disons tout de suite que ces produits de débitage ne forment pas un ensemble homogène tant du point de vue de leur module, de la technique de débitage, que de leur régularité. Ces disparités révèlent, en partie, leur appartenance à différentes phases de la chaîne opératoire.

Une mise en forme du nucléus par deux pseudo-crêtes postéro-latérales est identifiée grâce à de nombreuses lames et lamelles latérales caractéristiques. Néanmoins, quelques crêtes ou recoupes de crêtes antérieures sont présentes (une vingtaine ; fig. $6, \mathrm{n}^{\text {os }} 1$ et 2 ). Celles de petit module pourraient correspondre à de la réparation en cours de débitage, mais six éléments de plus gros module posent problème dans le cadre du schéma de mise en forme ci-dessus exposé (fig. $6, \mathrm{n}^{\text {os }} 1$ et 2 ). La future surface de débitage est installée sur une plage restée en partie corticale et/ou attestant une ou plusieurs plage(s) mate(s) : près d'une trentaine de lames et lamelles montre des plages corticales résiduelles ou des plages mates situées au centre de leur face supérieure et recoupées des deux côtés par des enlèvements débités après chauffe. Ce procédé fait référence à ce qui est connu en contexte chasséen récent sur les sites de Trets (Binder, 1984, p. 83, et fig. 5, $\mathrm{n}^{\circ} 6$ ) et de Lattes (Léa, 2002, p. 98, 343, et pl. $2, \mathrm{n}^{\circ} 10$, pl. $8, \mathrm{n}^{\text {os }} 7$ et 11$)$. De plus, quelques lames et lamelles débitées après chauffe ont une face supérieure entièrement mate. Ces éléments, pour la première fois identifiés sur le site des Trois Termes, correspondent à une ouverture de la surface de débitage après traitement thermique $^{3}$.

\section{STYLES DE DÉBITAGE ET ÉLÉMENTS DE DATATION}

Certains nucléus chauffés ont été débités sur place par pression comme l'attestent les lames et lamelles de plein débitage (environ 80), les lames et lamelles latérales et de réfection, ainsi que les 7 nucléus à lamelles. L'optimum de débitage (lamelles centrales de rythme 212' et 123/321; Binder, 1987) est néanmoins peu représenté (seulement un peu plus d'une trentaine d'éléments), et son importante fragmentation n'autorise pas une étude stylistique approfondie. Cette sous-représentation de l'optimum de débitage pourrait s'expliquer par le prélèvement, en contexte d'atelier, des éléments les plus réguliers.

Plusieurs indices permettent cependant de proposer, en accord avec les données céramiques, une datation assez récente pour cette industrie. Il s'agit de plans de pression inclinés (révélés par les talons inclinés des lamelles de plein débitage, et par les talons déjetés de plusieurs lamelles latérales), de réductions de la corniche par pression (néanmoins rares), et de 2 nucléus de type Trets (Binder, 1984, 1991 ; fig. 8). Ces derniers montrent 6 négatifs de lamelles dont le dernier est l'enlèvement central. L'étude du rythme de débitage atteste dans les deux cas l'obtention de lamelles de code 123/321. Un troisième nucléus est de « type mixte » (Léa, 1997, 2002, 2004) à savoir qu'il comporte à la fois des caractères évolués (plan de pression incliné) et des caractères plus archaïques (nervures convergentes). Dans l'assemblage, certains produits de débitage correspondent à un style de débitage plus semi-conique et pourraient être mis en relation avec ce type mixte (nous pensons notamment à des lamelles de plein débitage aux nervures convergentes et à des latérales très couchées sur le flanc). Les quatre autres nucléus à lamelles n'ont pas de style de débitage déterminé car ils ont fait l'objet de reprises. Sur trois d'entre eux, il est cependant encore possible d'identifier un plan de pression incliné.

3. Les négatifs mats de leur face supérieure correspondent soit à des enlèvements unidirectionnels, montrant que le débitage de produits laminaires plus ou moins réguliers a pu commencer avant la chauffe, soit à des enlèvements de préparation et des enlèvements unidirectionnels. 


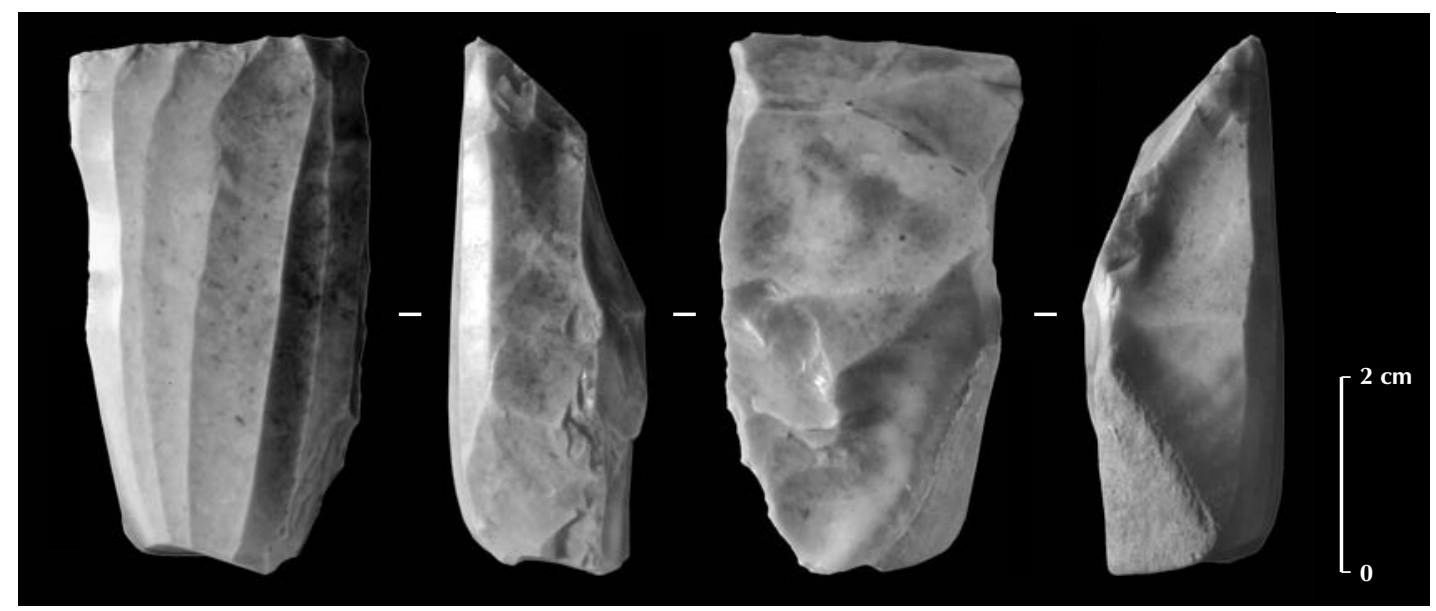

Fig. 8 - Les Trois Termes : nucléus de type Trets.

\section{PRODUCTIONS SPÉCIALISÉES ET PRODUCTIONS DOMESTIQUES}

Cette première approche de l'industrie des Trois Termes apporte d'ores et déjà des éléments nouveaux dans la connaissance des centres de production des préformes avant exportation.

L'hypothèse de la réalisation sur place du traitement thermique des préformes a été proposée à partir de l'identification de blocs éclatés par le feu ainsi que par celle de phases de mise en forme avant et après chauffe. Cette hypothèse nous semble cohérente avec celle de spécialistes assurant les premières phases de la chaîne opératoire. En effet, la chauffe de préformes nécessiterait en théorie un certain savoir-faire : réalisation d'un milieu pour la chauffe, élévation progressive de la température, maintien d'une température optimale pendant une durée déterminée, refroidissement progressif (Binder, Perlès, 1990).

Par ailleurs, les phases de mises en forme présentes aux Trois Termes révèlent un travail sans doute effectué en série, comme en témoigne le surprenant effectif d'éclats d'ouverture du plan de pression. Leur abondance révèle de même les quantités industrielles de préformes réalisées sur les sites producteurs. Ces préformes, sans doute exportées, sont rares dans l'assemblage étudié $(n=3)$. Nous en connaissions déjà un exemple sur le site du Bois de l'Acourt (Mormoiron, Vaucluse ; Binder, 1991, p. 264, fig. 2, n 3). Remarquons que sa morphologie cintrée et allongée correspond assez bien à celle d'une partie des préformes des Trois Termes, révélée par l'analyse des éclats d'ouverture du plan de pression (voir fig. 4, $\mathrm{n}^{\circ} 3$, p. 236 et fig. 5, $\mathrm{n}^{\text {os }} 1$ et 2 , p. 237).
Néanmoins, et c'est certainement l'un des aspects les plus intéressants, si la mise en forme des nucléus à lamelles a constitué l'activité principale des artisans du site des Trois Termes, elle n'a pas été la seule. Le débitage sur place de plusieurs nucléus à lamelles évoquerait, parallèlement à cette activité de spécialistes, une production répondant à leurs besoins quotidiens ou domestiques (fig. 8). Ceci permettrait alors de penser à une certaine durée d'installation. La présence de céramiques irait également dans le même sens (Léa et al., 2004b). L'outillage lithique retouché est pourtant rarissime : 2 petits perçoirs et 1 grattoir sur éclat. Cependant, l'utilisation au Chasséen de lamelles brutes étant assez fréquente (Gassin, 1996), une étude tracéologique est nécessaire pour vérifier l'utilisation sur place de ces produits de débitage.

Enfin, les productions spécialisées à haut niveau de savoir faire ne sont pas les seules attestées sur le site des Trois Termes. La présence de préformes non réussies, dont une est beaucoup trop chauffée, pourrait en effet être le résultat du travail de tailleurs moins expérimentés. Il en va de même d'une production d'éclats non normalisés et débités par percussion directe à partir de 17 nucléus. Ces nucléus sont de plusieurs sortes : il s'agit soit de nucléus en silex bédoulien chauffé (reprises d'anciens nucléus à lamelles $)^{4}$, soit de nucléus en silex non chauffé $(n=2)$, soit d'éclats thermiques repris. Néanmoins, rien ne s'oppose à ce que cette production d'éclats irréguliers corresponde à une production complémentaire de celle des lamelles

4. À l'heure actuelle, nous ne connaissons en effet aucun exemple de traitement thermique de préformes en vue d'un débitage d'éclats. 
débitées par pression, afin de répondre à des besoins d'autre nature.

\section{ROCALIBERT (PIOLENG) PRÉSENTATION DU SITE}

Ce gisement préhistorique de plein air est situé très près du Rhône (fig. 1), à un peu plus d'un kilomètre au sud-est du village de Piolenc, au pied du massif calcaire des collines de Cargaule, (Buisson-Catil et al., 1999). Les occupations chasséennes sont anciennement connues dans la région, notamment pour les riches sites des Roches et des Combes, grâce à de nombreuses prospections et à la réalisation de sondages et de fouilles sporadiques (Courtin, 1974, p. 74 et 87 ; Courtin, Gutherz, 1976, p. 352-369 ; Lavoinne, Parisi, 1981, p. 231 ; Sauzade, 1983, p. 26, 110 et 199 ; Devalque, 2000). L'obsidienne est bien attestée sur différents établissements (18 éléments en totalité) et les analyses effectuées sur 12 échantillons révèlent une provenance sarde du Monte Arci (Devalque, 2000, p. 100-102 et 244 ; Binder, Courtin, 1994).

Le mobilier lithique que nous présentons ici provient d'un sondage de deux mètres carrés réalisé par J. BuissonCatil à la suite de travaux clandestins (Buisson-Catil et al., 2000). Là encore ce qui surprend en premier lieu, c'est la concentration, pour l'heure inexpliquée, des vestiges lithiques (596 éléments) céramiques (695 tessons dont 69 bords et 30 préhensions) et fauniques (non comptabilisés). Les observations stratigraphiques n'ont malheureusement pas permis de comprendre le contexte archéologique en raison de l'exiguïté du sondage. Le niveau archéologique épais de 15 à $20 \mathrm{~cm}$ apparaît sous une couche stérile et repose sur un niveau argilo-sableux (BuissonCatil in Buisson-Catil et al., 2000, p. 3). Les objets ont été le plus souvent retrouvés en position horizontale ou subhorizontale.

Une première approche de la céramique a révélé une phase récente du Chasséen: présence de vases à épaulement, vases à col distinct et multiforés divers (Georjon in Buisson-Catil et al., 2000, p. 14-18). Plusieurs caractères permettent de préciser cette attribution et d'orienter les affinités géographiques (ibid.). L'association d'écuelles sinueuses, à cran, à paroi redressée et à paroi très évasée se retrouve dans le groupe $\mathrm{C}$ du Chasséen de la moyenne vallée du Rhône (Thiercelin, 1986). Les écuelles sinueuses pourraient rappeler des exemplaires du groupe de Lagozza
(Beeching, 1995) et notamment du site de l'Isolino di Varese (Guerreschi, 1977 ; Bertone, Fedele, 1991), alors qu'elles sont rares en Ligurie et absentes en Provence. Les écuelles à paroi très évasée, ou écuelles surbaissées, sont en revanche attestées en Provence (aven de Vauclare à Esparron-de-Verdon dans les Alpes-de-Haute-Provence); Trets dans les Bouches-du-Rhône (Courtin, 1974) ; grotte de l'Église, en couche 5, à Baudinard dans le Var (Courtin, 1967), etc. Les vases à épaulement étroit à haut col sont caractéristiques du Chasséen récent de la moyenne vallée du Rhône (groupe C et E de A. Beeching). Enfin, les vases globuleux à ouverture rétrécie, apparemment absents de la moyenne vallée du Rhône, sont présents sans bord ourlé en Languedoc: horizon 7 de Font-Juvénal (Guilaine et al., 1990), et sont fréquents avec un bord ourlé en Provence: grotte C et grotte de l'Église à Baudinard dans le Var (Courtin, 1967; Courtin, Pélouard, 1971), Escanin aux Baux-de-Provence (Montjardin, 1966, 1970), abri de Sous Ville à Correns dans le Var (Courtin, 1974). Enfin, quelques rares éléments céramiques $(\mathrm{n}=5)$ seraient par ailleurs attribuables au Néolithique final (Georjon in Buisson-Catil et al., 2000, p. 17).

\section{UNE EXCEPTIONNELLE CONCENTRATION DE NUGLÉUS}

L'industrie lithique, en excellent état de conservation, montre une exceptionnelle concentration de nucléus en silex bédouliens ( $\mathrm{n}=81$, soit 13,5\% de l'assemblage). À ces nucléus étaient associés 289 éclats $(48,5 \%)$ et 184 produits laminaires $(31 \%)$, auxquels il faut ajouter 42 éléments brûlés ou taillés à partir d'autres matières premières (calcédoine et autres silex indéterminés).

Deux productions différentes sont attestées sur silex bédouliens suivant l'intervention ou non du traitement thermique dans la chaîne opératoire.

- Les éléments en silex non chauffés sont peu nombreux ( $\mathrm{n}=39$, soit $6,5 \%)$ et principalement représentés par des lames et lamelles $(n=29)$ importées sous forme de supports et de produits finis. Il s'agit dans la presque totalité des cas de produits laminaires appartenant à la phase de plein débitage (hormis trois exceptions et quatre éléments indéterminés) dont le parallélisme des bords et des nervures est bon, et qui semblent très majoritairement débités par pression. Un nucléus repris en pièce esquillée et quelques éclats complètent cet ensemble d'éléments non chauffés. 


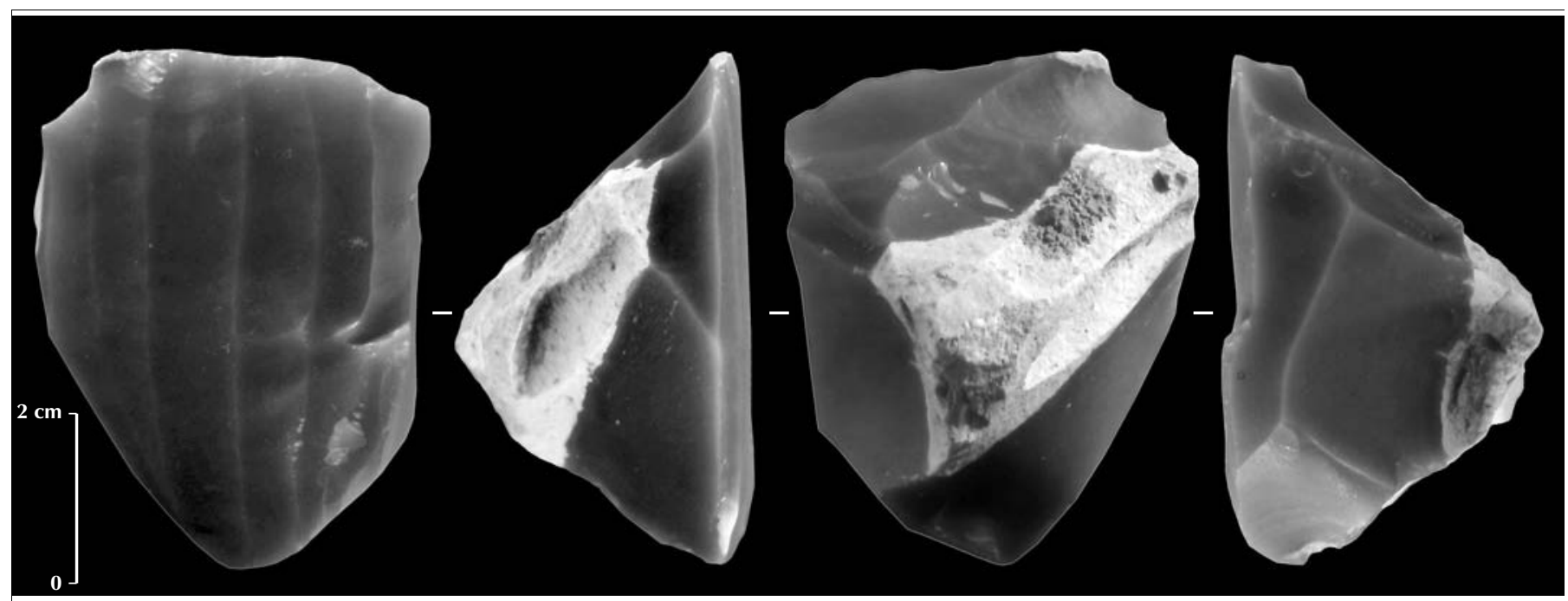

Fig. 9 - Rocalibert : nucléus de type Trets.

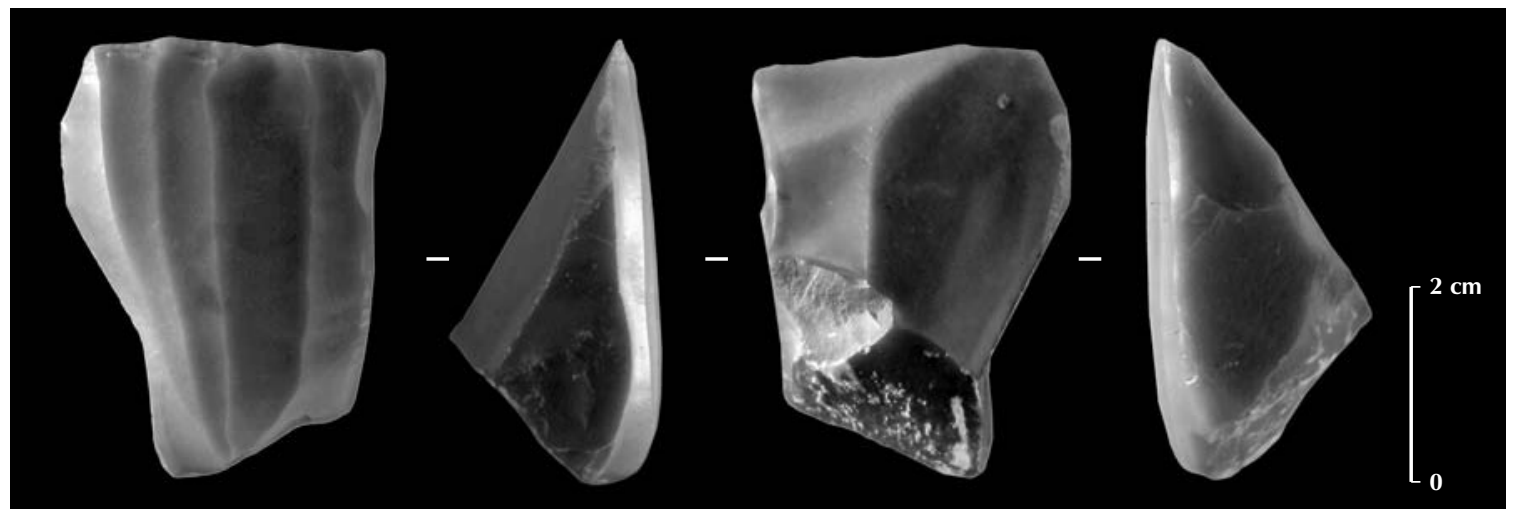

Fig. 10 - Rocalibert : nucléus de type Trets.

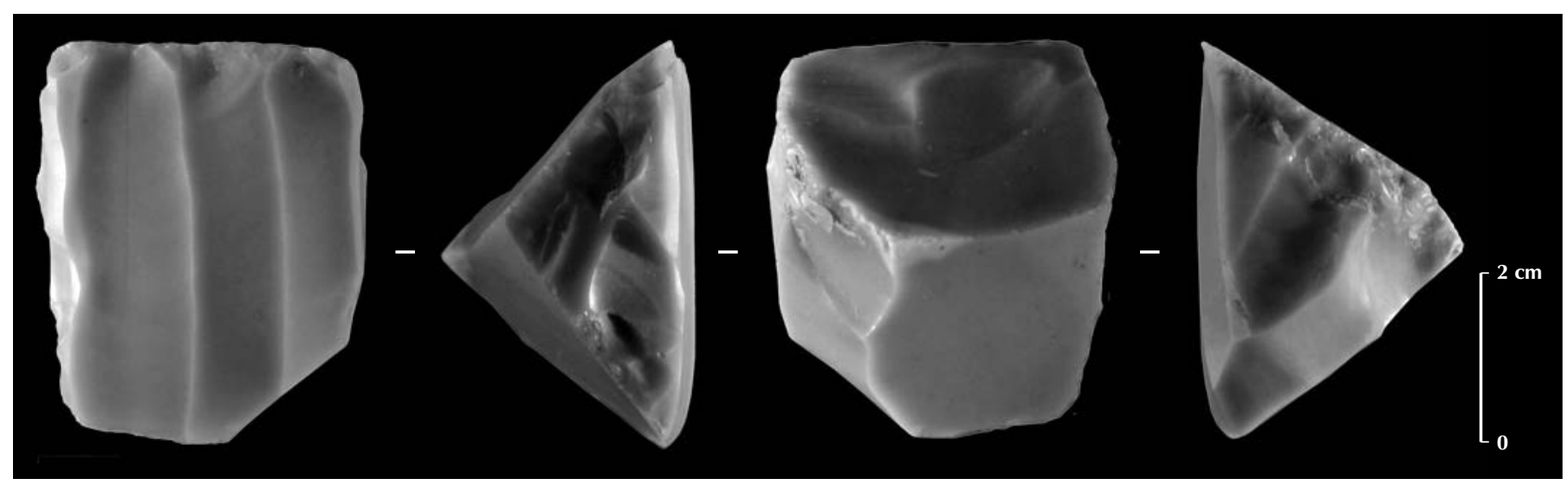

Fig. 11 - Rocalibert : nucléus de type Trets. 
- Le traitement thermique est clairement identifié sur la plupart des nucléus et des produits de débitage $(93,5 \%)$. Contrairement au site des Trois Termes, les nucléus chauffés sont arrivés à Rocalibert déjà préformés (absence d'éclats corticaux et de gros éclats de mise en forme). Néanmoins, l'identification de 5 éclats d'ouverture du plan de pression (dont un remonte sur le nucléus et montre sur sa face supérieure une plage mate ; fig. $4, \mathrm{n}^{\circ} 1$ ), suggérerait que la fin de la mise en forme ait pu en partie avoir lieu sur le site. Le débitage de lamelles sur place est bien attesté par la présence d'éclats de reprise $(\mathrm{n}=32)$ dont des tablettes de réfection du plan de pression de différents modules $(\mathrm{n}=12)$, ainsi que par celle de lamelles latérales et de réfection.

Cependant, le nombre de lamelles débitées après chauffe $(n=153)$ est totalement dérisoire par rapport à celui des nucléus $(n=80)$. La réalisation du rapport lamelles/nucléus donne le chiffre aberrant de moins de 2 lamelles par nucléus, alors que la production a dû avoisiner les 4000 lamelles en totalité, à raison de 50 lamelles par nucléus en moyenne. Il ne s'agit certes ici que d'un ordre de grandeur approximatif évalué par l'expérimentation du débitage par pression de nucléus en silex bédoulien de Murs (communication orale P.-J. Texier ; Texier in Gallet, 1998, p. 42). On pourrait objecter (et avec raison) que les dimensions réduites du sondage n'offrent sans doute pas un échantillon représentatif de l'industrie lithique du site. Il est néanmoins intéressant de constater que cette évaluation théorique du nombre de lamelles débitées à Rocalibert est bien supérieure aux effectifs jusque-là connus sur les sites consommateurs les mieux fournis, tels Lattes dans l'Hérault (2 390 lamelles; Léa, 2002, p. 95 et 125), La Cabre dans le Var (près de 2000 lamelles ; Léa, 1997, p. 39 et 2003a), et Auriac secteur P. IV dans l'Aude (698 lamelles ; Briois, 1997, p. 317). Ces constatations permettent alors de suggérer (avec toutes les réserves que le contexte de découverte impose) une exportation, depuis le site de Rocalibert vers les sites consommateurs, de lamelles débitées après chauffe. Cette hypothèse est confortée par la faible représentation de lamelles appartenant à la phase du plein débitage (une cinquantaine environ dont seulement 34 lamelles centrales de code 212' ou 123/321). Les éléments de l'optimum de débitage (212' et $123 / 321$ ) ne sont d'ailleurs pas toujours très réguliers (en raison de nervures sinueuses notamment) et l'on peut envisager une sélection des supports les plus réguliers pour l'exportation.

\section{VARIABILITÉ DES STYLES DE DÉBITAGE}

L'étude de cet exceptionnel ensemble de nucléus chauffés met en évidence une certaine variabilité dans les styles de débitage (Léa in Buisson-Catil et al., 2000).

Le style de débitage chasséen récent (type de Trets; Binder, 1991) est représenté par 24 individus (fig. 9 à 11). Ces nucléus sont mis en forme par deux pseudo-crêtes postéro-latérales dont les négatifs de préparation sont débités depuis le plan de pression lui-même (Binder, 1984, p. 83), et aussi parfois, depuis l'extrémité distale du nucléus. De section quadrangulaire, ils ont une surface de débitage plate et un plan de pression en général lisse, incliné voire très incliné, qui constitue dans certains cas la totalité de la face postérieure du nucléus. Les plages mates peuvent concerner les flancs, le plan de pression et/ou la face postérieure ; des surfaces corticales résiduelles sont parfois visibles sur le dos du nucléus. Les négatifs de lamelles montrent des terminaisons obtuses. Enfin, ce débitage plat, manquant parfois trop de convexité, a pu provoquer des réfléchis $(\mathrm{n}=8$ sur les nucléus). La variabilité des modules observée au sein de ce groupe de nucléus révèle que certains d'entre eux ont bien été débités selon le mode quadrangulaire jusqu'à la fin de la chaîne opératoire (le plus grand mesure $5,5 \mathrm{~cm}$ de hauteur pour plus de $4 \mathrm{~cm}$ de largeur; les deux plus petits mesurent moins de $2,5 \mathrm{~cm}$ de hauteur pour une largeur d'environ 1,8 cm). Ce style récent se retrouve sur une partie des lamelles de l'assemblage: certaines lamelles de plein débitage ont un talon lisse incliné et certaines lamelles débordantes un talon déjeté ; leur corniche est réduite par pression et/ou est abrasée selon les cas.

Les autres nucléus sortent du schéma précédemment décrit. Il s'agit tout d'abord de nucléus à plan de pression incliné mais dont l'extrémité distale est aiguë et montre des enlèvements convergents ( $\mathrm{n}=13$; fig. 12). L'un d'eux est

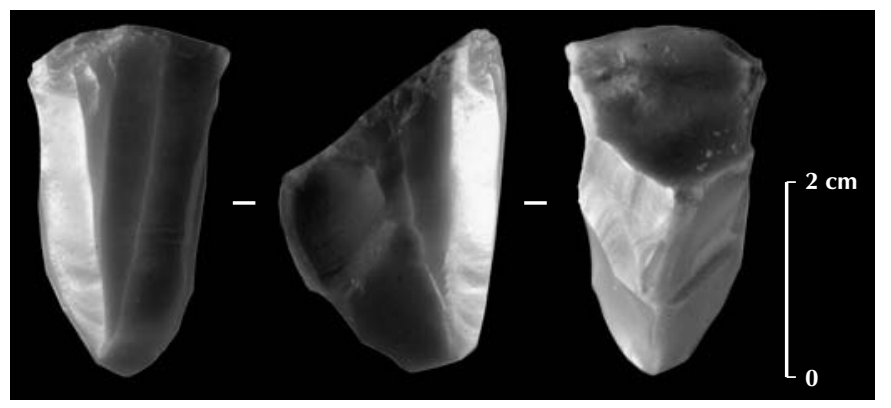

Fig. 12 - Rocalibert : nucléus de type mixte (semi-conique à plan de pression incliné). 


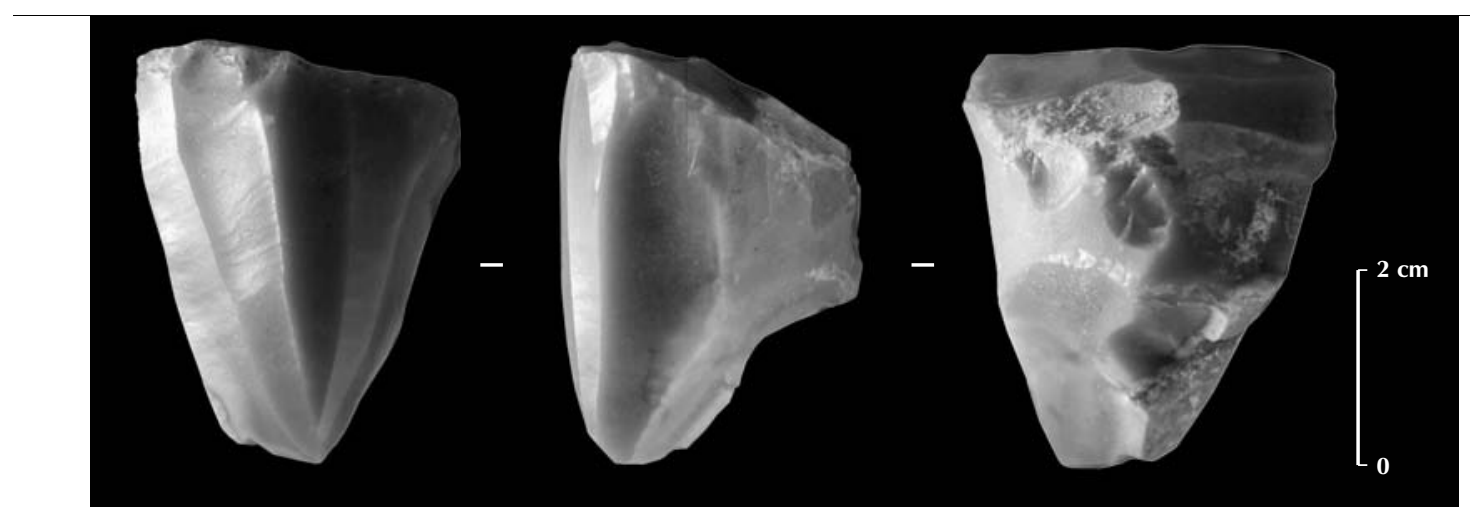

Fig. 13 - Rocalibert : nucléus semi-conique à plan de pression non incliné.

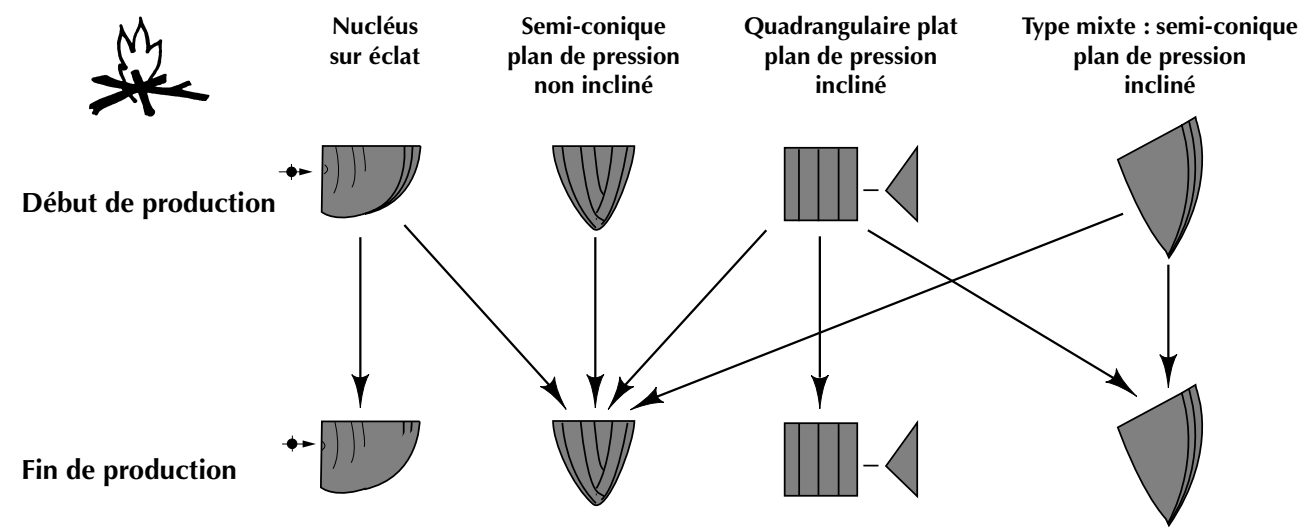

Fig. 14 - Transformation du nucléus en cours de production.

sur éclat. Ces nucléus, dont la section peut-être quadrangulaire ou triangulaire $(n=8)$, seraient d'un " type mixte » (voir fig. 12, p. 243 ; Léa, 2002, 2004). Ils font référence à des nucléus déjà mentionnés en Languedoc oriental comme sur les sites du Mas Rouge et de Lattes (Hérault) en contexte chasséen récent (Léa, 2002, 2004). Il s'agit ensuite de deux nucléus semi-coniques à plan de pression facetté non incliné et de section subcirculaire (fig. 13). Les négatifs des lamelles montrent une forte convergence en partie distale. Certains produits de débitage et d'entretien sont à mettre en relation avec ce style de débitage: lamelles à nervures convergentes, tablettes d'avivage du plan de pression (dans un débitage de type Trets, elles sont en effet rarissimes), et petits éclats d'entretien pour éviter les outrepassages. Ces éclats, pour la première fois identifiés en contexte chasséen, sont débités depuis la face postérieure du nucléus et emportent une partie de l'extrémité distale, correspondant alors à l'enlèvement d'un pyramidion (Tixier, 1963). Enfin, il faut noter la présence d'un nucléus de style original en contexte chasséen, qui se distingue totalement des groupes ci-dessus décrits. Ce nucléus sur éclat allongé (5 cm de longueur) et étroit (1,8 cm d'épaisseur) montre deux surfaces de débitage lamellaire situées sur deux plans opposés, mais débitées depuis le même plan de pression. Il peut rappeler, sans être strictement identique, un exemplaire de la Bobilà Madurell en Catalogne, qui n'est cependant pas sur silex bédoulien (Gibaja, Terradas, 2001, p. 44).

Comment expliquer la présence de différents styles de débitage (fig. 14) ? L'identification d'un style de débitage semi-conique associé à un style de débitage quadrangulaire plat (type de Trets) remet-elle en question l'homogénéité chronologique de l'assemblage (Binder, 1984, 1991) ? Le style de débitage semi-conique (plan de pression non incliné et extrémités distales aiguës) ne nous semble pas suffisamment représenté ici (seulement 2 nucléus) pour faire référence à celui connu dans le Chasséen ancien de la grotte de l'église supérieure (couches 7 et 8 ) et de Fontbrégoua 
(couches 19-24) ${ }^{5}$. De plus, les talons facettés des lamelles, caractéristiques du style ancien, sont ici quasi absents. Rappelons enfin que la céramique n'indiquait pas non plus deux différentes phases d'occupation. En ce qui concerne les nucléus de type mixte, deux hypothèses peuvent être posées : soit ils correspondent à une variabilité du débitage de type Trets (les nucléus deviennent semi-coniques en cours ou en fin de production ; fig. 14), soit ils révèlent une morphologie particulière de préformes et trahissent alors une certaine diversité des préformes issues des centres de production. Ces deux hypothèses pourraient cependant ne pas être exclusives. En effet, sur le site de Lattes par exemple les deux phénomènes sont attestés : d'une part, différents types de préformes chauffées sont identifiés (nucléus sur éclat, nucléus semi-coniques à plan de pression incliné ou non, nucléus quadrangulaires plats), et, d'autre part, le passage d'un nucléus quadrangulaire plat à un nucléus semiconique en fin de production a été démontré (Léa, 2002, 2004). Dans l'assemblage qui nous intéresse ici, nous n'avons pas pour l'instant les éléments qui permettent de trancher en faveur de l'une ou l'autre de ces hypothèses.

À Rocalibert, les résultats de l'examen des styles de débitage ont néanmoins été limités par la fréquente reprise des nucléus pour un débitage d'éclats, ainsi que par la transformation du nucléus en cours de débitage. Celle-ci est révélée grâce à l'observation de nucléus attestant deux surfaces de débitage consécutives. Après transformation, le débitage de lamelles a pu continuer à partir du même plan de pression (mais sur une autre face du nucléus), à partir d'un plan de pression opposé aux dépens de la même surface de débitage ou aux dépens d'une autre, ou bien à partir d'un plan de pression perpendiculaire au plan de pression initial (surfaces de débitage croisées ; fig. 15).

Ces deux procédés - reprise pour un débitage d'éclats et transformations du nucléus - permettent une exploitation maximum du volume de matière première. Or, ceci peut paraître totalement paradoxal dans la mesure où le site de Rocalibert se trouve relativement près des gîtes de silex bédouliens. Le site voisin des Combes (Piolenc) a de même livré plusieurs nucléus complètement épuisés (collection de C. Devalque). Ces remarques rejoignent celles formulées à partir du gisement chasséen des Martins à Roussillon éloigné de seulement 6 km de Murs (Renault, 1990, p. 30).

5. Seuls plusieurs croisements de données établis à partir des lamelles 212' et 123/321, trop peu nombreuses ici, permettraient de valider la présence de deux phases chronologiques (Léa, 1997, 2002, 2003b, 2004).

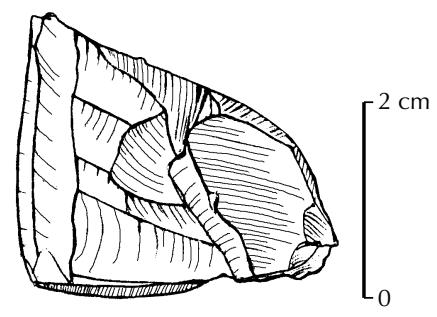

Fig. 15 - Rocalibert : nucléus attestant deux surfaces de débitage consécutives et orthogonales (échelle 1/1).

L'épuisement des nucléus ne peut pas s'expliquer par l'éloignement des sources de matière première, mais révèlerait plutôt le statut particulier du silex bédoulien, et ce, même dans l'aire de production.

\section{IMPLICATION DANS LA COMPRÉHENSION DU FONCTIONNEMENT DES RÉSEAUX DE DIFFUSION DES SILEX BÉDOULIENS}

La remarquable série de Rocalibert apporte ainsi des éléments nouveaux à notre connaissance de l'organisation des réseaux de diffusion. Elle permet de plus de proposer plusieurs hypothèses concernant différentes échelles.

- À l'échelle du site, cette inhabituelle concentration de nucléus traduit la présence d'un atelier. L'outillage retouché est rarissime dans l'assemblage: 1 pièce bifaciale brûlée, 1 pièce esquillée et 5 grattoirs. Des fouilles d'envergure sont néanmoins indispensables pour remettre en contexte les vestiges mis au jour et appréhender les activités qui s’y sont déroulées ainsi que l'organisation spatiale du site.

- Du point de vue des productions lithiques, la variabilité des styles de débitage a été mise en relief, dans un contexte où l'homogénéité chronologique des vestiges ne peut être mise en doute ni par l'étude du lithique ni par celle de la céramique. Ceci pourrait traduire une certaine variabilité du débitage lamellaire et/ou une diversité des productions fabriquées sur les gîtes d'extraction et de mise en forme. Ces observations font alors référence à l'une des questions posées en introduction, à savoir si la diversité des préformes révèle un approvisionnement multiple c'est-à-dire à partir de plusieurs centres de production, ou bien si à un unique centre de production correspondent plusieurs types de préformes (Léa et al., 2004b). De futures recherches sur les centres de production devraient permettre de répondre à ces questions.

- À l'échelle des réseaux de circulation des productions en silex bédouliens, Rocalibert semble occuper une place particulière. L'exportation de lamelles régulières en silex bédouliens, depuis Rocalibert vers les sites consommateurs, 
est en effet suggérée par le très grand déficit de celles-ci par rapport aux nucléus. Rappelons que c'est la première fois qu'une telle concentration de nucléus est découverte en contexte chasséen. Des lamelles débitées après chauffe circuleraient donc sous forme de supports à l'instar des lames robustes en silex non chauffé dont la diffusion est depuis longtemps connue (Binder, Gassin, 1988). Cette hypothèse trouve un argument de poids avec la présence, sur certains sites consommateurs, de lamelles chauffées dont le débitage sur place n'est pas attesté, comme c'est par exemple le cas à la grotte des Arene Candide, Ligurie, en contexte chasséen (Binder, 1998, p. 124-126). De plus, il semble bien que certains sites consommateurs, éloignés des centres de production, montrent à l'inverse un déficit de nucléus par rapport au nombre de lamelles. Tel est le cas du site de Lattes (2 390 lamelles chauffées pour seulement 11 nucléus ; Léa, 2002, p. 95,125, Léa, 2004) ou d'Auriac secteur P.IV (698 lamelles chauffées pour 5 nucléus ; Briois, 1997, p. 317). Les nucléus seraient aussi assez rares sur les grands sites de plaine de la Drôme (Beeching, communication orale). Dans le cadre de cette réflexion, il est alors intéressant de faire mention de l'ancienne découverte sur l'oppidum de Sainte-Luce (Vercoiran, Drôme) d'un étui, aménagé dans un os long de bovidé, contenant plusieurs lamelles en silex blond (Gras, 1976, p. 72, fig. 1), car il nous offre un exemple des modalités de transport et/ou de stockage de ces supports somme toute très fragiles ${ }^{6}$. La diffusion de lamelles débitées après chauffe pourrait d'ailleurs être beaucoup plus fréquente que ce que l'on pense, mais elle n'est pas souvent identifiable : elle passe en effet totalement inaperçue dans un assemblage où le débitage sur place de préformes chauffées est attesté.

\section{SYNTHÈSE ET HYPOTHÈSES}

Les résultats préliminaires de l'étude de ces deux sites du Chasséen récent, les Trois Termes et Rocalibert, apportent déjà quelques informations concernant les problématiques exposées en introduction. Ils permettent de mettre en évidence différents aspects de la production et la diffusion des produits.

6. Notons toutefois qu'une expérimentation menée par B. Gassin, avec un os long de bovidé, a montré que les lamelles étaient gravement endommagées (Gassin, communication personnelle). Il faudrait donc envisager simultanément l'emploi de tissus pour protéger efficacement ces fragiles supports dont le tranchant s'effiloche facilement.
- Sur les sites des Trois Termes et de Rocalibert deux ateliers de taille spécialisés, jouant un rôle dans la fabrication ou la diffusion des productions en silex bédouliens, sont identifiés (fig. 16). Les activités de taille, présentes sur l'un et l'autre atelier, ne sont pas identiques : elles illustrent des fonctions de site différentes ainsi que des aspects de la production pour partie complémentaires. Les deux ateliers n'occupent en effet pas la même place au sein des réseaux de circulation de silex bédouliens (fig. 16). La phase de mise en forme des préformes chauffées est bien représentée sur le site des Trois Termes alors qu'elle est totalement absente du site de Rocalibert (seules quelques rares ouvertures du plan de pression pourraient avoir été débitées sur ce dernier site). Le site de Rocalibert est donc totalement dépendant des réseaux de circulation des préformes chauffées et de ceux des lames non chauffées, dont il n'assure pas la production. En ce sens Rocalibert est un site récepteur au contraire du site des Trois Termes. Notons par ailleurs que, dans l'assemblage des Trois Termes, aucun élément en obsidienne ou en silex rubané n'a été identifié contrairement à celui de Rocalibert.

- Le fonctionnement des réseaux de diffusion de silex bédouliens atteste une certaine complexité. La circulation des matériaux pourrait en effet ne pas être linéaire, des sites producteurs aux sites consommateurs. À Rocalibert, les nucléus chauffés sont arrivés préformés, et ont été débités sur place avant l'exportation d'une partie au moins des lamelles les plus régulières. D’une manière générale, on peut alors envisager l'existence de sites en marge de l'aire de production, qui joueraient le rôle de «places centrales » de matières premières (Renfrew, 1984). Ils seraient en quelque sorte des ateliers relais ou redistributeurs, entre sites producteurs et sites consommateurs. Dans le cas de Rocalibert, cette hypothèse est de même confortée par l'importante présence d'éléments en obsidienne. Cette idée de sites redistributeurs n'est certes pas nouvelle puisqu'elle avait déjà été proposée pour l'atelier spécialisé de la Cabre (Var), riche en silex bédouliens comme en obsidienne (Léa, 1997, 2003a), ainsi que pour certains autres sites du Languedoc (Léa, 2002, p. 30-31). Rocalibert offre donc un argument supplémentaire en ce sens.

- Un site producteur peut-il aussi être un site consommateur? Les Trois Termes et Rocalibert sont tous deux des sites producteurs de biens voués, en partie au moins, à l'exportation après transformation (fig. 16) : le site des Trois Termes exporte des préformes prêtes à débiter, et celui de Rocalibert des supports (lamelles débitées par pression sur 


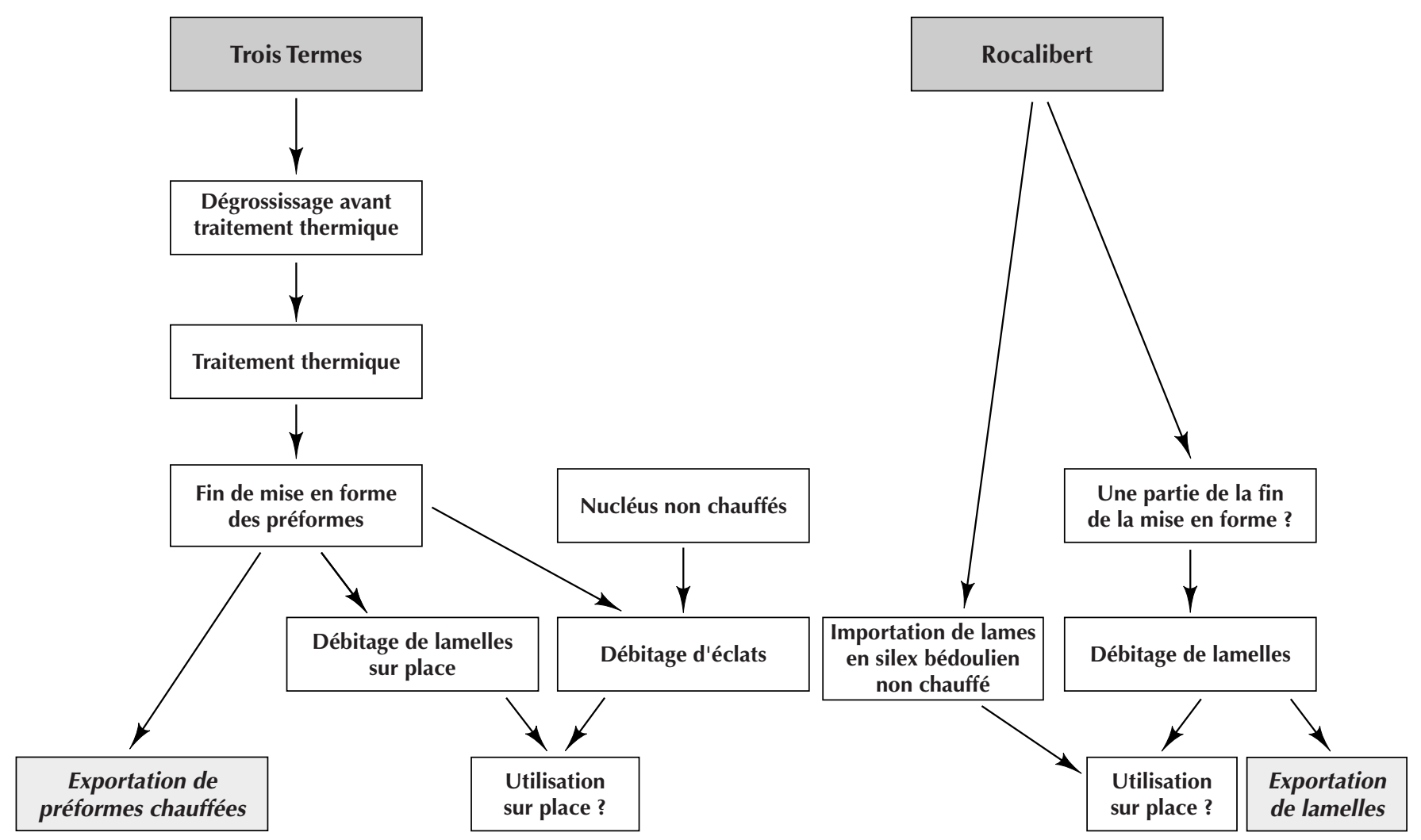

Fig. 16 - Étapes de la chaîne opératoire représentées aux Trois Termes et à Rocalibert.

le site à partir de préformes chauffées). Mais ces deux sites pourraient aussi être des sites partiellement consommateurs (Léa, sous presse a ; Léa et al., 2004b). Aux Trois Termes, le débitage de lamelles est bien attesté parallèlement au travail de mise en forme des préformes. Remarquons néanmoins que, sur les deux sites, l'outillage retouché est rarissime et rappelle en cela les contextes miniers vosgiens de mise en forme de haches polies en pélites-quartz (Pétrequin, Jeunesse, 1995, p. 56). Cependant, comme nous l'avons déjà évoqué, au Chasséen les lamelles sont souvent utilisées brutes (Gassin, 1996) et des éléments céramiques sont présents aux Trois Termes et à Rocalibert. Il faudra donc vérifier, grâce à une étude tracéologique, l'utilisation ou la non-utilisation des lamelles brutes et, le cas échéant, caractériser la nature des activités qui se seraient déroulées sur place. Dans quelle mesure révèlent-elles des activités à usage domestique? Ces réflexions rejoignent alors certaines des questions posées en introduction et concernant le contexte de production. Dans le cadre de cette problématique, les analyses technologiques et tracéologiques seules ne sont de toute façon pas à même d'apporter les réponses. Il est bien évident que la réalisation de fouilles et l'interaction des différents types d'études (géomorphologiques, céramiques, fauniques, etc.), sont ici indispensables à la compréhension du fonctionnement de ces sites.

- Plusieurs niveaux de savoir-faire seraient attestés sur les centres de production. Un très haut niveau de savoir-faire est certes bien représenté par les phases de mise en forme aux Trois Termes. L'hypothèse d'un travail effectué en série a de plus été posée en raison de l'étonnant effectif des ouvertures du plan de pression. Parallèlement à cela, quelques éléments témoigneraient de l'activité de tailleurs moins expérimentés. Cette hypothèse fait alors référence à ce qui est connu sur les ateliers vosgiens de mise en forme des haches, où sont identifiées des ébauches approximatives (Pétrequin, Jeunesse, 1995, p. 71, 72).

$$
\text { * * }
$$

Ces résultats préliminaires mettent en évidence une certaine complexité dans l'organisation de la production et dans la diffusion des silex bédouliens en Vaucluse. Cette complexité fait en partie écho à celle qui caractérise les 
réseaux de circulation en dehors de l'aire de production (Léa et al., 2004a). Les analyses technologiques des assemblages lithiques des sites consommateurs éloignés des centres de production ont en effet déjà montré une grande diversité dans l'alimentation de ces sites (Briois et al., 1998 ; Léa, 2002, p. 386-387 ; 2004). Cette diversité concerne, comme nous l'avons énoncé en introduction, la nature des productions exportées, leur quantité, mais aussi les modalités d'utilisation : par exemple, une même préforme chauffée peut être débitée en un temps et en un lieu (Binder, Gassin, 1988, p. 121), ou bien être débitée en différents temps et différents lieux (Léa, 2002, p. 392-393 et sous presse b). L'observation de ces phénomènes suggérerait une hiérarchisation des réseaux, sans doute organisés en réseaux principaux - à une échelle interrégionale du Vaucluse jusqu'aux marges de l'aire de diffusion - et en réseaux secondaires - à une échelle locale, au sein d'un terroir - (Léa et al., 2004a). Dans ce contexte, l'interaction entre les données concernant les sites producteurs, redistributeurs et consommateurs, à différents niveaux du réseau, est indispensable à la compréhension de ce système de diffusion des silex bédouliens au Chasséen dans sa globalité.

\section{Remerciements}

Je tiens sincèrement à remercier plusieurs personnes qui ont contribué à la réalisation de cet article : M. Grenet qui a réalisé les planches de dessins ici illustrées ; J. Buisson-Catil, J. Vaquer et D. Binder qui ont bien voulu effectuer une première relecture ; C. Georjon qui a examiné la céramique des Trois Termes et de Rocalibert et qui m'a fait part de ses observations; P.-J. Texier pour d'intéressantes informations; M. Castan et C. Devalque pour avoir accepté de mettre à ma disposition leurs collections. 


\section{BIBLIOGRAPHIE}

\section{BARTHELÉMY A}

1952-1956 : « Recherches archéologiques dans la région de Veaux à Malaucène (Vaucluse) : la grotte du Levant de Léaunier et les industries des stations voisines », Bulletin de la Société des sciences naturelles de Vaucluse, 23-27, p. 41-55.

\section{BEECHING A.}

1995 : « Nouveau regard sur le Néolithique ancien et moyen du Bassin rhodanien ", in VoruZ J.-L. (ÉD.) Chronologies néolithiques: de 6000 à 2000 avant notre ère dans le Bassin rhodanien, Actes des $\mathrm{XI}^{e}$ rencontres sur le Néolithique de la région RhôneAlpes, Ambérieu-en-Bugey, 19-20 sept. 1992, éd. Société préhistorique rhodanienne, p. 93-112.

Bertone A., Fedele $\mathbf{F}$.

1991: « Découvertes récentes dans la vallée de Susa et le problème des relations avec le Chasséen ", in BeEching A., Binder D., Blanchet J.-C. et al. (ÉD.) Identité $d u$ Chasséen, Actes du Colloque international de Nemours, 1989, Nemours, éd. APRAIF (Mémoires du musée de Préhistoire d'Îlede-France, 4), p. 69-78.

BINDER D.

1984 : "Systèmes de débitage laminaire par pression: exemples chasséens provençaux ", in TiXIER J., INIZAN M.-L., ROCHE H. (DIR.) Préhistoire de la pierre taillée -2Économie du débitage laminaire, Paris, Publications du Cercle de recherches et d'études préhistoriques, p. 71-84.

1987 : Le Néolithique ancien provençal. Typologie et technologie des outillages lithiques, Paris, éd. du CNRS (Suppl. à Gallia Préhistoire, XXIV), $209 \mathrm{p}$.

1991 : Facteurs de variabilité des outillages lithiques chasséens dans le sud-est de la France, in Beeching A., Binder D., Blanchet J.-C. et al. (ÉD.), Identité du Chasséen, Actes du Colloque international de Nemours, 1989, Nemours, éd. APRAIF (Mémoires du musée de Préhistoire d'Îlede-France, 4), p. 261-272.

1998 : Silex blond et complexité des assemblages lithiques dans le Néolithique liguro-provençal, in D'ANNA A., BINDER D. (ÉD.), Production et Identité culturelle Rencontres méridionales de Préhistoire récente, Actes de la deuxième session, Arles, 8-9 nov. 1996, Antibes, Association pour la promotion et la diffusion des recherches archéologiques, p. 111-128.
Binder D., avec la collab. de Barbier M., GUILBERT R.

1998 : Rapport de synthèse sur les prospections thématiques en Provence rhodanienne 1995-1997, Recensement des disponibilités en matières premières lithiques dans la région Provence-Alpes-Côte d'Azur, UMR 6636 du CNRS, Université de Provence - Ministère de la Culture et de la Communication.

Binder D., COURTIN J.

1994 : « Un point sur la circulation de l'obsidienne dans le domaine provençal ", Gallia Préhistoire, 36, p. 310-322.

Binder D., Gassin B.

1988 : « Le débitage laminaire chasséen après chauffe: technologie et traces d'utilisation », in BEYRIES S. (ÉD.), Industries lithiques, tracéologie et technologie, Oxford, British Archaeological Reports, International Series, 411, p. 93-125.

Binder D., Perlès C. avec la collab. de INIZAN M.-L., LECHEVALLIER M.

1990 : « Stratégies de gestion des outillages lithiques au Néolithique ", Paléo, 2, p. 257-283.

BRIOIS F.

1997 : Les industries lithiques du Languedoc méditerranéen (6000-2000 av. J.-C.). Rythmes et évolution dans la fabrication des outillages de pierre taillée néolithiques entre mer et continent, thèse de doctorat, École des hautes études en sciences sociales, $557 \mathrm{p}$.

Briois F., Brossier S., Gernigon K., Vaquer J.

1998 : " Polymorphisme des industries chasséennes en silex entre le Rhône et l'Aquitaine ", in D'ANnA A., Binder D. (ÉD.) Production et Identité culturelle, Rencontres méridionales de Préhistoire récente, Actes de la deuxième session, Arles (Bouches-du-Rhône) 8-9 nov. 1996, Antibes, Association pour la promotion et la diffusion des recherches archéologiques, p. 129-144.

Buisson-Catil J., Georjon C., Léa V.

1999 : Le site de Rocalibert (Piolenc, Vaucluse), Bilan scientifique, Direction régionale des Affaires culturelles Provence-Alpes-Côte d'Azur, 21, p. 188-190.

2000 : Le gisement Néolithique moyen de Rocalibert à Piolenc (Vaucluse), Rapport de sondages (nov. 2000), Ministère de la Culture et de la Communication, Direction régionale des Affaires culturelles, Service régional de l'Archéologie Provence-Alpes-Côte d'Azur.
CARIAS L.

1919 : « Sur un maillet à rainure en roche importée trouvé à Murs », Rhodania, p. $492-495$.

Catelan A., Catelan L.

1922a : " La grotte du Levant de Léaunier, Malaucène, continuation des fouilles », Association française pour l'avancement des Sciences, Congrès de Montpellier, p. 490-492.

1922b : " La grotte du Levant de Léaunier, Malaucène, continuation des fouilles ", Association française pour l'avancement des Sciences, Congrès de Montpellier, p. $425-431$.

CotTe V.

1924 : Documents sur la Préhistoire de Provence Stations néolithiques et protohistoriques, Aixen-Provence, Librairie d'histoire de la Provence A. Dragon, vol. III.

CourTIN J.

1967 : « La grotte de l'Église à Baudinard (Var) », Gallia Préhistoire, 10, 2, p. 282-300.

1974 : Le Néolithique de la Provence, Paris, Klincksieck (Mémoires de la Société préhistorique française, 11), $360 \mathrm{p}$.

Courtin J., GUtherz X.

1976 : « Les bracelets de pierre du Néolithique méridional ", Bulletin de la Société préhistorique française, 73, p. 352-369. Courtin J., Pelouard S.

1971 : « Un habitat chasséen en HauteProvence: "la grotte C" de Baudinard (Var) ", Bulletin de la Société préhistorique française, 68 , 2, p. 540-566.

DÉCHELETTE J.

1908 : Manuel d'archéologie, Paris, Librairie des Archives nationales et de la Société de l'École des Chartes, $750 \mathrm{p}$.

Devaloue C.

2000 : Piolenc... avant Piolenc - L'archéologie de la cité des origines à l'an mil, Piolenc, éd. Le Félibre.

DEYDIER M.

1904 : « Les maillets de Murs », Bulletin de la Société préhistorique française, I, p. $167-174$.

1905 : «A propos des maillets de Malaucène », Bulletin de la Société préhistorique française, II, p. 138-139 et 217-219.

1907 : «Contribution à l'étude des maillets et haches préhistoriques ", Revue préhistorique, 8 .

1908 : « Le préhistorique aux environs du mont Ventoux, région Sud-Ouest 
( $1^{\mathrm{re}}$ partie) ", in Congrès préhistorique de France, Compte rendu de la troisième session, Autun, 1907, Paris, éd. Schleicher Frères, p. 135-171.

1911 : «Le préhistorique aux environs du mont Ventoux, région Sud-Ouest (2 $2^{\mathrm{e}}$ partie) ", in Congrès préhistorique de France, Compte rendu de la sixième session, Tours, 1910, Paris, Société préhistorique de France, p. 196-226.

\section{Gallet M.}

1998 : Pour une technologie des débitages laminaires préhistoriques, Paris, CNRS Éditions, $177 \mathrm{p}$.

GASSIN B.

1996 : Évolution socio-économique dans le Chasséen de la grotte de l'Église supérieure (Var) : apport de l'analyse fonctionnelle des industries lithiques, Paris, CNRS Éditions (Monographies du CRA, 17), 327 p.

Gibaja J.-F., Terradas X.

2001 : El tratamiento térmico en la producción lítica : el ejemplo del neolítico medio catalán, Cypsela, 13, p. 29-56.

GRAS R.

1976 : «Oppidum de Sainte-Luce, Vercoiran (Drôme) ", in BocQueT A., LAGRAND C. (ÉD.), Néolithique et Âge des métaux dans les Alpes francaises, IX $X^{e}$ congrès de l'UISPP, 13-18 sept. 1976, Livret guide de l'excursion A9, Nice, UISPP, p. 65-71.

GUERRESCHI G.

1977 : « L'Isolino di Varese, insediamento préistorico », Sibrium, XIII, p. 520.

Guilaine J., Amiel C., Barthès P., Coularou J., Vaquer J.

1990 : «Le chasséen de l'abri de FontJuvénal ", in Guilaine J., Gutherz X. (DIR.), Autour de Jean Arnal, Montpellier, éd. Recherches sur les premières communautés paysannes, p. 163-175.

GUILBERT R.

2001 : «"Le Sansonnet” et "les Agnels" (Vaucluse), un exemple de fragmentation thermique intentionnelle du silex au Sauveterrien », Paléo, 13, p. 245-250.

Inizan M.-L., Reduron M., Roche H. TIXIER J.

1995 : Technologie de la pierre taillée, Meudon, Publications du Cercle de recherches et d'études préhistoriques, 4, 199 p.

Lavoinne M.-L., Parisi S., Camps G., CampsFABRER $\mathbf{H}$.

1981 : Atlas préhistorique du Midi méditerranéen Feuille d'Orange, Paris, éd. du CNRS, 273 p.

LÉA V.

1997 : Contribution à l'étude des sites de plein air chasséens: étude technologique de l'industrie lithique en silex blonds du site de la Cabre (Var), mém. de maîtrise, univ. de Provence (centre d'Aix), 203 p.

2002 : Les industries lithiques du Chasséen en Languedoc oriental : caractérisation par l'étude technologique, thèse de doctorat, univ. de Provence (Aix-Marseille-I), $465 \mathrm{p}$.

2003a : «Mesurer, quantifier et croiser. Une méthode d'approche pour les industries lithiques du Chasséen méridional ", Histoire et Mesure, XVIII, 1-2, p. 3-39.

2003b : «Un atelier de fabrication de microperçoirs au Chasséen : le site de la Cabre (Var) ", Bulletin de la Société préhistorique française, 100, 3, p. 517-532.

2004 : Les industries lithiques du Chasséen en Languedoc oriental : caractérisation par l'étude technologique, British Archaeological Reports, 1232, 215 p., 73 pl.

sous presse a : «Raw, Pre-heated or ready to use : discovering specialist supply systems for flint industries in Mid-Neolithic (Chasseen) communities in southern France », Antiquity, 78, n 302.

sous presse b : «Quelles identités pour l'industrie lithique du Néolithique moyen de Montou ? », in Claustre F. (ÉD.), La grotte de Montou (P.-O.): étude chronostratigraphique, environnementale et fonctionnelle, Paris, éd. de la Maison des sciences de l'Homme (Documents d'archéologie française)

Léa V., Gassin B., Briois F.

2004a: «Fonctionnement des réseaux de diffusion des silex bédouliens du $\mathrm{V}^{\mathrm{e}}$ au $\mathrm{IV}^{\mathrm{e}}$ millénaire: questions ouvertes ", in Dartevelle H. (COORD.), Ve Rencontres méridionales de Préhistoire récente, ClermontFerrand, nov. 2002, Préhistoire du SudOuest, supplément $\mathrm{n}^{\circ} 9$, p. 405-420.

LÉa V., Georjon C., Lepère C., Sénépart I. Thirault E.

2004b : «Chasséen vauclusien qui es-tu ? », in Buisson-Catil J., Guilcher A., Hussy C., Pagny M., Olive M. (DIR.), Vaucluse préhistorique : le territoire, les hommes, les cultures et les sites, Avignon, éd. Barthélemy, p. 165-200.

MONTJARDIN R.

1966 : « Le gisement néolithique d’Escanin aux Baux-de-Provence ", Cahiers rhodaniens, 13, p. 5-103.

1970 : « Le gisement néolithique d'Escanin aux Baux-de-Provence ", Cahiers rhodaniens, 15 , p. 5-152.

Moulin F.

1905 : « À propos du gisement à maillets de
Malaucène ", Bulletin de la Société préhistorique francaise, 1905 , p. 72 et 169.

1908 : «Contribution à l'étude des exploitations préhistoriques de silex avec maillets de pierre en Provence. Note préliminaire relative au détail des carrières ", Revue préhistorique, I, p. 10-16.

Pétrequin P., Jeunesse C. (DIR.)

1995 : Carrières vosgiennes et échanges de lames polies pendant le Néolithique (5400-2100 av. J.-C.), Paris, éd. Errance, 127 p.

\section{RAYMOND P.}

1905 : «Les maillets de Malaucène (Vaucluse), puits d'extraction et taillerie de silex néolithiques ", Bulletin de la Société préhistorique francaise, II, p. 17-26.

\section{Renault S.}

1990 : « Le gisement de plein air des Martins, Roussillon, Vaucluse. Étude de l'industrie lithique ", in CHENORKIAN R. (DIR.), Travaux du Laboratoire d'anthropologie et de préhistoire des pays de la Méditerranée occidentale, univ. de Provence, LAPMO, p. 27-48.

\section{Renfrew C.}

1984 : «Trade as action at distance ", in RENFREW C. (ÉD.), Approaches to social archaelogy, Cambridge, Harvard University Press, p. 86-134.

\section{SAuzade G.}

1983 : «Les sépultures du Vaucluse du Néolithique à l'âge du Bronze ", Études quaternaires, 6, p. 249 p.

\section{ScHMID E.}

1962 : « Vom Silex-Bergbau bei VeauxMalaucène in Sud-Frankreich », Der Anschnitt, 15, 3, Bochum, p. 10-21.

\section{THIERCELIN F.}

1986 : « La céramique chasséenne de SaintUze (Drôme) ", in Actes des Rencontres Néolithique Rhône-Alpes, 1986, 2, p. 33-40.

TIXIER J.

1963 : Typologie de l'Épipaléolithique du Maghreb, Alger, Mémoires du Centre de recherches anthropologiques préhistoriques et ethnographiques.

VAQUER J.

1990 : Le Néolithique en Languedoc occidental, Paris, éd. du CNRS, 398 p.

Vayson de Pradenne A.

1923 : « Les maillets préhistoriques de Murs et de Malaucène ", L'Anthropologie, 1923, XXXIII, p. 171.

1934 : «L'industrie des ateliers à maillets de Murs ", in Congrès préhistorique de France, Compte rendu de la dixième session, Nîmes-Avignon, 1931, Paris, bureaux de la Société préhistorique française, p. 146-179. 